\title{
WAPO-A1 is the causal gene of the 7AL QTL for spikelet number per spike in wheat
}

\author{
Saarah Kuzay $\oplus^{1 \neq}$, Huiqiong Lin $\oplus^{1,2 \ddagger}$, Chengxia Li $\oplus^{1,2}$, Shisheng Chen $\oplus^{1,3}$, Daniel \\ P. Woods $\mathbb{D}^{1,2}$, Junli Zhang $\mathbb{1}^{1}$, Tianyu Lan $\mathbb{D}^{4}$, Maria von Korff ${ }^{4,5}$, Jorge Dubcovsky $\mathbb{D}^{1,2 *}$ \\ 1 Department of Plant Sciences, University of California, Davis, California, United States of America, \\ 2 Howard Hughes Medical Institute, Chevy Chase, Maryland, United States of America, 3 Peking University \\ Institute of Advanced Agricultural Sciences, Weifang, Shandong, China, 4 Institute for Plant Genetics, \\ Heinrich Heine University, Duesseldorf, Germany, 5 Cluster of Excellence on Plant Sciences "SMART Plants \\ for Tomorrow's Needs", Heinrich Heine University, Duesseldorf, Germany
}

$\ddagger$ SK and HL contributed equally to this work.

* jdubcovsky@ucdavis.edu

\section{OPEN ACCESS}

Citation: Kuzay S, Lin H, Li C, Chen S, Woods DP, Zhang J, et al. (2022) WAPO-A1 is the causal gene of the 7AL QTL for spikelet number per spike in wheat. PLoS Genet 18(1): e1009747. https://doi. org/10.1371/journal.pgen.1009747

Editor: Sarah Hake, "USDA-ARS Pacific West Area", UNITED STATES

Received: July 26, 2021

Accepted: December 18, 2021

Published: January 13, 2022

Peer Review History: PLOS recognizes the benefits of transparency in the peer review process; therefore, we enable the publication of all of the content of peer review and author responses alongside final, published articles. The editorial history of this article is available here: https://doi.org/10.1371/journal.pgen.1009747

Copyright: @ 2022 Kuzay et al. This is an open access article distributed under the terms of the Creative Commons Attribution License, which permits unrestricted use, distribution, and reproduction in any medium, provided the original author and source are credited.

Data Availability Statement: We deposited the Kronos near isogenic line with the $\mathrm{H} 2$ introgression in the National Small Grains Collection (PI 698810). All data are presented in the text and supplementary materials. The raw data for all

\section{Abstract}

Improving our understanding of the genes regulating grain yield can contribute to the development of more productive wheat varieties. Previously, a highly significant QTL affecting spikelet number per spike (SNS), grain number per spike (GNS) and grain yield was detected on chromosome arm 7AL in multiple genome-wide association studies. Using a high-resolution genetic map, we established that the A-genome homeolog of WHEAT ORTHOLOG OF APO1 (WAPO-A1) was a leading candidate gene for this QTL. Using mutants and transgenic plants, we demonstrate in this study that WAPO-A1 is the causal gene underpinning this QTL. Loss-of-function mutants wapo- $A 1$ and wapo-B1 showed reduced SNS in tetraploid wheat, and the effect was exacerbated in wapo1 combining both mutations. By contrast, spikes of transgenic wheat plants carrying extra copies of WAPO$A 1$ driven by its native promoter had higher SNS, a more compact spike apical region and a smaller terminal spikelet than the wild type. Taken together, these results indicate that WAPO1 affects SNS by regulating the timing of terminal spikelet formation. Both transgenic and wapo1 mutant plants showed a wide range of floral abnormalities, indicating additional roles of WAPO1 on wheat floral development. Previously, we found three widespread haplotypes in the $\mathrm{QTL}$ region ( $\mathrm{H} 1, \mathrm{H} 2$ and $\mathrm{H} 3)$, each associated with particular WAPO-A1 alleles. Results from this and our previous study show that the WAPO-A1 allele in the $\mathrm{H} 1$ haplotype (115-bp deletion in the promoter) is expressed at significantly lower levels in the developing spikes than the alleles in the $\mathrm{H} 2$ and $\mathrm{H} 3$ haplotypes, resulting in reduced SNS. Field experiments also showed that the $\mathrm{H} 2$ haplotype is associated with the strongest effects in increasing SNS and GNS $(\mathrm{H} 2>\mathrm{H} 3>\mathrm{H} 1)$. The $\mathrm{H} 2$ haplotype is already present in most modern common wheat varieties but is rare in durum wheat, where it might be particularly useful to improve grain yield. 
figures and Supplemental Tables are available in S1 Data file.

Funding: JD received support for this project from the Agriculture and Food Research Initiative Competitive Grants 2017-67007-25939 (WheatCAP), USDA National Institute of Food and Agriculture (NIFA, https://nifa.usda.gov/) and from the Howard Hughes Medical Institute (https://www. hhmi.org/). The USDA-NIFA grant supported the salaries of SK, JZ and SC. The Howard Hughes Medical Institute supported the salaries of JD, $\mathrm{HL}$, CL and DW. DW is a Howard Hughes Medical Institute Fellow of the Life Sciences Research Foundation (http://www.lsrf.org/) that paid his salary for three years. The funders had no role in study design, data collection and analysis, decision to publish, or preparation of the manuscript.

Competing interests: The authors have declared that no competing interests exist.

\section{Author summary}

A region on wheat chromosome 7A has been previously shown to affect the number of spikelets and grains per spike as well as total grain yield in multiple breeding programs. In this study, we show that loss-of-function mutations in the WAPO1 gene located within this region reduce the number of spikelets per spike and that additional transgenic copies of this gene increase this number. These results demonstrate that WAPO1 is the gene responsible for the differences in grain number and yield associated with the 7A chromosome region. Among the three main variants identified for this gene, we demonstrate in field experiments that the $\mathrm{H} 2$ variant is associated with the largest increases in number of spikelets and grains per spike. The H2 WAPO1 variant is frequent in bread wheat breeding programs but is almost absent in modern pasta wheat varieties. Therefore, the introgression of the $\mathrm{H} 2$ represents a promising opportunity to improve grain yield in pasta wheat.

\section{Introduction}

Wheat is an essential staple crop for global food security. It is highly adapted to a wide variety of climates and production systems, and provides more than $20 \%$ of the calories and protein consumed by the human population [1]. Although further increases in grain yield are required to feed a continuously growing population, historical yield trend studies have shown a decrease in the relative rates of grain yield gains in some wheat growing regions [2]. This has prompted new efforts to understand and improve the productivity of both common (Triticum aestivum, genomes AABBDD) and durum wheat (T. turgidum ssp. durum, genomes AABB).

Identifying genes controlling total grain yield is challenging due to its complex quantitative nature and genotype by environment interactions [3]. However, grain yield can be dissected into more discrete yield components with higher heritability. Total grain yield can be partitioned into several yield components, including number of spikes per area unit, spikelet number per spike (SNS), grain number per spikelet, and average grain weight. Among these traits, SNS usually exhibits high heritability because it is established early in the reproductive phase when the terminal spikelet is formed [4], limiting the effect of environmental conditions after this point.

A highly significant and stable QTL for SNS was identified on chromosome arm 7AL in multiple genome-wide association studies (GWAS) including a panel of soft red winter wheats in the US [5], panels of European winter wheats [6-9], a panel of US and CIMMYT photoperiod-insensitive spring wheats, and six biparental populations which comprised different wheat market classes [10]. In our previous study, we generated two high-resolution genetic maps to delimit this SNS QTL to an 87-kb region $(674,019,191-674,106,327 \mathrm{bp}$, RefSeq v1.1) containing four candidate genes [10]. Among these genes, we identified TraesCS7A02G481600 as the most promising candidate gene, based on the presence of a non-synonymous polymorphism that co-segregated with SNS in biparental populations segregating for different haplotypes in the candidate region [10].

The wheat gene TraesCS7A02G481600 is orthologous to the Oryza sativa (rice) gene ABERRANT PANICLE ORGANIZATION1 (APO1), hence it was designated as WHEAT ORTHOLOG of APO1 (WAPO1). Loss-of-function mutants in rice APO1 reduce panicle branching and spikelet number [11], supporting $W A P O-A 1$ as a promising candidate gene for the SNS QTL $[7,8,10]$. The rice APO1 gene and its homolog in Arabidopsis thaliana (Arabidopsis), UNUSUAL FLORAL ORGANS (UFO), encode an F-box protein that is a component of an SCF 
(Skp1-Cullin-F-box-protein) ubiquitin ligase [12,13]. This domain is important to maintain the activity of LEAFY (LFY), a transcription factor that plays key roles in flowering and floral development [14].

In rice, mutations in $A P O 1$ or $L F Y$ (also known as $A P O 2$ and $R F L$ in rice) result in reductions in the number of branches and spikelets per panicle. The effect is similar in the apol lfy double mutant, suggesting that these two genes act cooperatively to control this trait [15]. Mutations in these two genes are also associated with floral abnormalities, with more severe phenotypes in the apo1 lfy double mutant than in either of the two single mutants. These results suggest that these two genes also play important roles in floral development [15]. Floral defects in the rice apol and Arabidopsis ufo mutants are concentrated to the internal floral whorls $[12,13]$.

Rapid changes in WAPO-A1 allele frequencies during wheat domestication and breeding suggest that this region is relevant to wheat improvement [10]. Three major haplotypes were identified in the 87-kb candidate gene region- $\mathrm{H} 1, \mathrm{H} 2$, and $\mathrm{H} 3$-each of which associated with different WAPO-A1 alleles. Haplotype $\mathrm{H} 3$ includes the ancestral alleles Wapo-A1c and Wapo$A 1 d$, which differ from each other by two synonymous substitutions, two SNPs in the single intron and one in the promoter, which likely have limited effect on gene function [10]. Haplotype $\mathrm{H} 3$ is present in the diploid donor of the A genome (T. urartu), cultivated emmer (T. turgidum ssp. dicoccon) and wild emmer (T. turgidum ssp. dicoccoides), and at low frequency in modern durum and common wheat varieties. Haplotype H1, present in over $99 \%$ of modern durum wheat varieties, has the Wapo-A1 $a$ allele that is characterized by a 115-bp deletion in the promoter and a change from aspartic acid to asparagine at position $384(\mathrm{D} 384 \mathrm{~N})$. This amino acid change is predicted to have a limited effect on protein structure and function (Table 1, BLOSUM62 score = 1). WAPO-A1 haplotype $\mathrm{H} 2$, the most frequent haplotype in modern common wheat varieties, carries the Wapo-Alb allele and differs from the ancestral haplotype by a cysteine to a phenylalanine polymorphism at amino acid position 47 (C47F) in a conserved region of the F-box motif $[7,8,10]$. This amino acid change is predicted to have a strong effect on protein structure and function (Table 1, BLOSUM62 score $=-2$ ). Linkage analysis in six different biparental populations established that the $\mathrm{H} 2$ haplotype was associated with higher SNS than both the $\mathrm{H} 1$ and $\mathrm{H} 3$ haplotypes [10].

Our previous study established WAPO-A1 as the best candidate gene for the 7AL SNS QTL [10], but functional validation was missing. In this study, we demonstrate that WAPO1 is the

Table 1. Comparison of regulatory and coding WAPO-A1 regions in wild type Kronos (H1 haplotype) and the three genomic constructs used in the transgenic plants.

\begin{tabular}{l|l|c|c|c|c|c|c}
\hline \multicolumn{1}{c|}{$\begin{array}{l}\text { RAPO-A1 haplotype } \\
\text { RefSeqv1.1 }\end{array}$} & $\begin{array}{l}\text { DNA } \\
\text { change }\end{array}$ & Protein effect & BLOSUM 62 score ${ }^{\text {a }}$ & Kronos & TmDV92 & LDN-C47 & LDN-F47 \\
\hline Promoter & & & & & & \\
\hline $674,080,862$ & 115 bp del & none & n/a & present & absent & absent & absent \\
\hline Coding Region & & & & & \\
\hline $674,081,601$ & G140T & C47F & -2 & C & C & C \\
\hline $674,081,843$ & C382G & P128A & -1 & P & A & P \\
\hline $674,082,413$ & A952G & Q273R & 1 & Q & R & Q & P \\
\hline $674,082,673674,082,674$ & G1212A C1213T & A360M & -1 & A & M & A \\
\hline $674,082,745$ & A1284G & N384D & 1 & N & D & D \\
\hline
\end{tabular}

a The more negative the BLOSUM 62 score is, the most likely is the change to affect protein structure or function.

${ }^{\mathrm{b}}$ This construct includes the edited F47 polymorphism in LDN-C47, but none of the promoter SNPs present in the natural haplotype H2 [10]. 
gene underpinning the SNS QTL by characterizing loss-of-function mutants and transgenic plants and exploring its spatial and temporal distribution in the developing spike. We also describe the flower abnormalities observed in plants with complete loss of WAPO1 activity and in transgenic plants with additional WAPO1 genes. Finally, we characterize the effect of different natural WAPO-A1 alleles on the number of spikelets and grains per spike and discuss their potential applications in common and durum wheat breeding programs.

\section{Results}

\section{Loss-of-function EMS and CRISPR mutations in WAPO1 reduce spikelet number per spike}

A search of the sequenced EMS-mutagenized population of tetraploid wheat Kronos [16], which carries the WAPO-A1 $\mathrm{H} 1$ haplotype, yielded one mutant line with a premature stop codon in WAPO-A1, but no stop codon or splicing site mutations were detected in the WAPO-B1 homeolog. The WAPO-A1 mutation W216* identified in Kronos mutant line K4222 results in a premature stop codon and the truncation of $51 \%$ of the encoded protein, which has 440 amino acids. The truncated region includes sequences highly conserved among grasses, suggesting that the truncated protein is either non-functional or has very reduced activity. The modified activity of the W216* truncated gene was confirmed by the significant SNS reduction in lines carrying the wapo-A1 mutant allele relative to those carrying the wild type (WT) allele in three different experiments (Fig 1). In the greenhouse experiment, the average $\mathrm{SNS}$ of $\mathrm{F}_{3}$ lines homozygous for wapo-A1 EMS mutant was 1.7 spikelets lower (14\% reduction, $P<0.001$ ) than the sister lines homozygous for the WT allele (Fig 1A). A similar reduction of 1.4 spikelets ( $7.3 \%$ reduction, $P<0.001$ ) was observed in a field experiment using homozygous $\mathrm{F}_{4}$ sister lines for the wapo-Al and WT alleles (Fig 1B). The number of florets per spikelet was not affected in the mutants.

To reduce potential variability originated from background mutations and more accurately determine the effect of the W216* truncation, we crossed the K4222 mutant to WT Kronos twice and selected homozygous $\mathrm{BC}_{1} \mathrm{~F}_{2}$ sister lines for an additional field evaluation using their $\mathrm{BC}_{1} \mathrm{~F}_{3}$ grains. The $\mathrm{BC}_{1} \mathrm{~F}_{3}$ plants homozygous for wapo- $\mathrm{A} 1$ had an average of 1.1 fewer spikelets per spike relative to the WT sister line (6.0\% reduction, $P=0.0035$, Fig 1C). Taken together, these three experiments indicate that loss-of-function of the A-genome homeolog of WAPO1 is sufficient to significantly reduce SNS.

To test the effect of the WAPO-B1 homeolog and validate the results for the EMS induced wapo-A1 mutant, we generated three independent $\mathrm{T}_{0}$ CRISPR-Cas9 transgenic Kronos plants with a guide RNA targeting both homeologs (S1 Table). We identified one line with a "T" insertion at position 510 from the ATG (4-bp upstream of the CGG PAM site) in both $W A P O-A 1$ and $W A P O-B 1$, and selected it for further characterization. This frameshift insertion alters $61.4 \%$ of the protein sequence (starting from amino acid 171), likely resulting in loss-of-function of both WAPO1 homeologs. We genotyped $110 \mathrm{~T}_{1}$ plants derived from the selected transgenic event using the WAPO-A1 and WAPO-B1 CAPS markers described in S1 Table. The proportion of lines homozygous for the WT Wapo-A1 (2.7\%) and/or Wapo-B1 (4.5\%) alleles was significantly lower than the expected $25 \%$, suggesting continuous CRISPR editing. This hypothesis was validated by the identification of a novel 5-bp frame-shift deletion starting at position 505 in WAPO-B1 (4-bp upstream of the PAM site) in line $\mathrm{T}_{1}-40$, which was re-sequenced and crossed to WT Kronos to eliminate the CRISPR-Cas9 transgene.

We created lines homozygous for all four possible combinations of the WAPO1 homeologs-WT, wapo-A1, wapo-B1, and wapo1 double mutant-by selecting $\mathrm{F}_{2}$ progeny derived from an $\mathrm{F}_{1}$ plant without the CRISPR-Cas 9 transgene. In a growth chamber experiment, we 

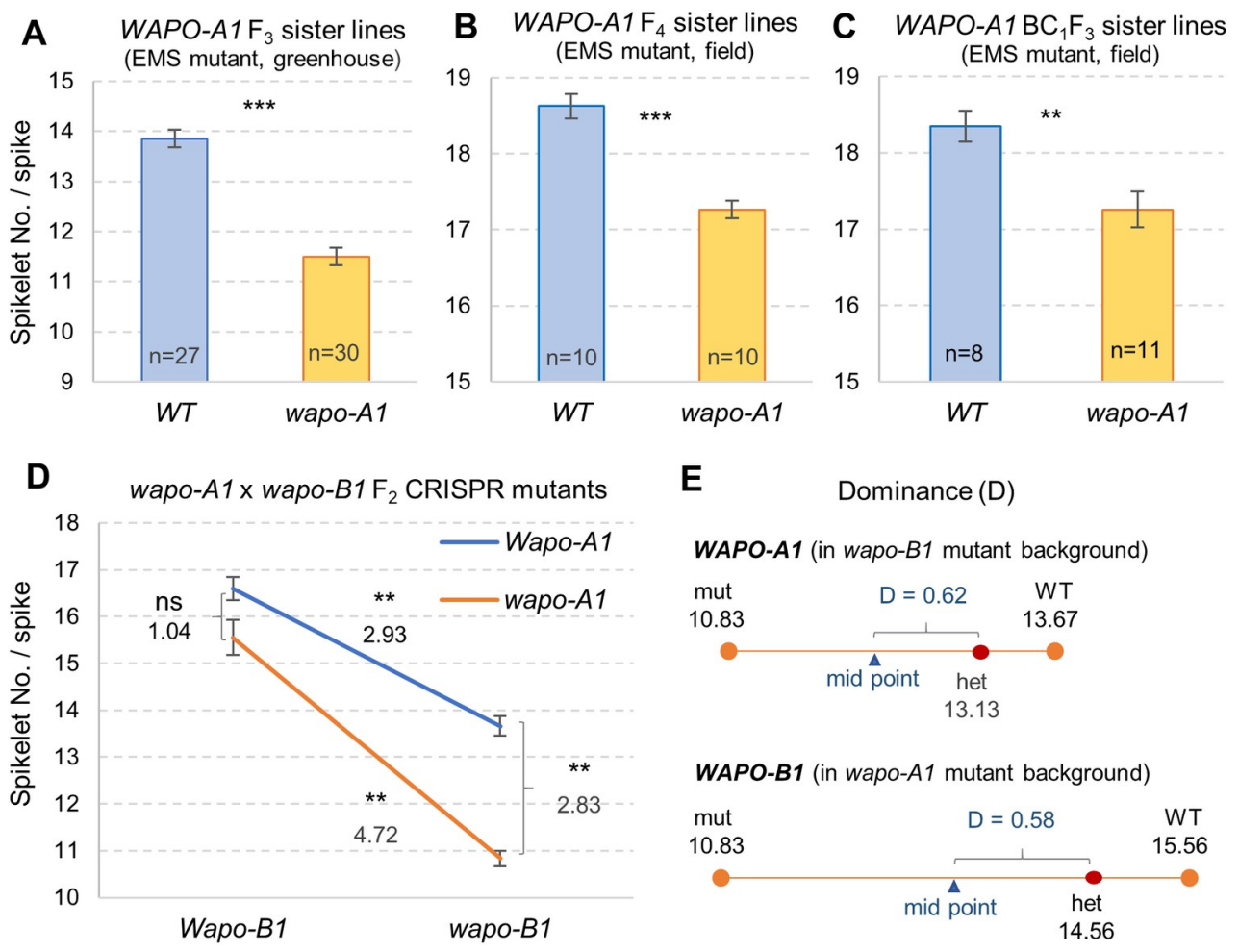

$\mathbf{E}$

Dominance (D)

WAPO-A1 (in wapo-B1 mutant background)
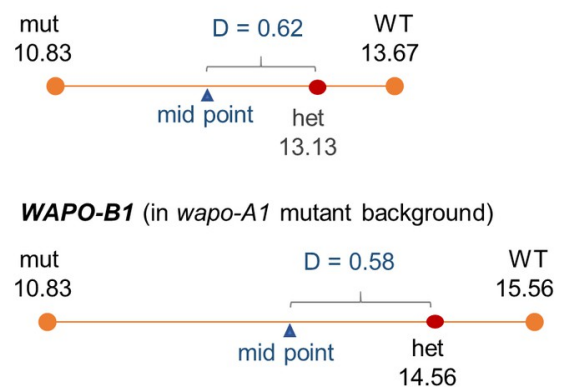

Fig 1. Differences in spikelet number per spike (SNS) in WAPO1 mutant lines. (A-C) Homozygous Kronos sister lines segregating for the EMS induced wapo-A1 W216* mutation. (A) $\mathrm{F}_{3}$ sister lines evaluated in the greenhouse. (B) $\mathrm{F}_{4}$ sister lines evaluated in the field. (C) $\mathrm{BC}_{1} \mathrm{~F}_{3}$ sister lines evaluated in the field. (D) Loss-of-function CRISPR induced wapo- $A 1$ and wapo-B1 mutations. Interaction graph showing the effect of wapo-A1 and wapo-B1 in tetraploid wheat Kronos plants without the CRISPR-Cas9 transgene. (E) Degree of dominance [17] for WAPO-A1 (in wapo-B1 background) and WAPO-B1 (in wapo-A1 background). Bars are average SNS and error bars are s.e.m. ns = not significant, ${ }^{*}=P<0.05,{ }^{* *}=P<0.01$ and $^{* * *}=P<0.001$. Data and statistical analyses used for this figure are available in Data A in S1 Data and panel A in S2 Table.

https://doi.org/10.1371/journal.pgen.1009747.g001

detected highly significant differences for SNS (panel A in S2 Table), but non-significant differences for heading date (panel B in S2 Table) or leaf number (panel C in S2 Table). Both wapo-A1 and wapo-B1 mutants showed significantly lower SNS than the WT (Fig 1D, panel A in S2 Table and Data A in S1 Data), with larger reductions in wapo-B1 (3 spikelets) than in wapo-A1 (1 spikelet). The transcript levels of the two homeologs were also different, with higher levels of WAPO-B1 than WAPO-A1 at different stages of spike development (S1 Fig). The effect of the wapo-A1 allele on SNS was highest when the B-genome homeolog was homozygous for wapo- $B 1$, and vice versa, resulting in a highly significant interaction $(P=0.0096$, Fig $1 \mathrm{D}$, and panel A in S2 Table). Partial dominance at the WAPO-A1 $(\mathrm{D}=0.61)$ and $W A P O-B 1(\mathrm{D}=0.58)$ loci (Fig $1 \mathrm{E})$ indicates partial functional redundancy between the two copies within each of the WAPO1 homeologs.

\section{Transgenic plants with additional WAPO1 copies show delayed heading time and higher SNS}

To compare the effect of three WAPO-A1 alleles encoding proteins with different non-synonymous polymorphisms (Table 1), we transformed three different constructs into Kronos. We used the bacterial artificial chromosome (BAC) libraries of diploid T. monococcum (accession DV92) [18] and tetraploid wheat Langdon (LDN-C47) [19] to clone the genomic regions of 
$W A P O-A 1$ from these two species. The cloned regions included the intron and regulatory regions (see Materials and Methods). We then used site-directed mutagenesis to change the cysteine at position 47 in LDN-C47 (H3 haplotype) into a phenylalanine (LDN-F47), resulting in a protein identical to the one encoded by the Wapo-A1b allele in the $\mathrm{H} 2$ haplotype (Table 1).

We transformed the three constructs into Kronos and obtained four independent transgenic events for TmDV92, three for LDN-C47, and five for the LDN-F47 derived allele, and evaluated their corresponding $T_{1}$ progeny in greenhouse experiments for SNS (Fig 2). Transgenic plants for all three constructs showed similar increases in SNS relative to the WT: LDN-C47 (13.4\%), TmDV92 (15.1\%) and LDN-F47 (15.5\%) (Fig 2). Increases in SNS were significant for two TmDV92 events, one LDN-C47 event (Fig 2A) and four LDN-F47 independent events relative to the WT control (Fig 2C).

The effects of the transgenes on heading date were smaller and slightly less significant than those for SNS. For heading date, a 7.0\% delay was observed in transgenic plants with the LDN-C47 construct, a 5.4\% delay for TmDV92, and a 6.3\% delay for LDN-F47 (Fig 2B and 2D). Heading date was positively and significantly correlated with SNS among the $\mathrm{T}_{1}$ lines segregating for each of the three constructs $(R=0.53-0.87, P<0.001$, Data B in S1 Data). Taken together, these results demonstrate that the presence of additional copies of WAPO1 in the transgenic plants increases SNS and delays heading date.

\section{CRISPR wapo1 mutant shows altered floral morphology}

In addition to the reduced SNS, wapo1 double mutant displayed a great diversity of floral abnormalities and produced a limited number of grains. To establish the frequency of the different floral abnormalities, we dissected 91 florets from 32 spikelets located at different positions along the spike from 14 different wapo1 mutant plants (Data C in S1 Data). While glumes, lemmas and paleae were normal, multiple abnormalities were observed in other floral organs. The number of lodicules, which is normally two per floret, varied from zero to four (average 1.95, Fig 3A). Moreover, lodicules were frequently fused with stamens (19.1\%), leaflike tissue (22.5\%) or both (5.6\%, Fig 3B-3D). The number of stamens per floret was lower than three in $42 \%$ of the florets (Fig 3A-3D), resulting in an average of 2.15 stamens per floret. Stamens were frequently fused with each other or with ovaries (6.6\%), lodicules (18.9\%), leaflike tissue $(9.2 \%)$ or combinations of two of the previous three categories (4.6\%, Fig 3B and 3C). Most florets had one pistil with one ovary, but $43 \%$ of the florets had multiple pistils, likely due to loss of floret meristem determinacy. In $28.4 \%$ of the florets, the ovaries were fused with leafy tissue (Fig 3D and Data C in S1 Data). For comparison, a wild-type Kronos floret is shown in Fig 4.

Since most of the floral abnormalities in wapo1 were detected in the internal whorls (lodicules, stamens and pistils), we explored the effect of the wapo1 mutant on the expression of class-B, - $C$ and -E MADS-box floral genes in developing spikes at the stamen primordia stage. Relative to the WT, the wapol mutant showed a significant down regulation of class-B (AP3-1 and PI1) and class-C (AG1 and AG2) MADS-box genes (Fig $3 \mathrm{E}$ and $3 \mathrm{~F}$ ), but no significant differences were detected for the control SEPALLATA MADS-box genes SEP1-2, SEP1-4, SEP1-6, or SEP3-2 (Fig 3G).

\section{WAPO1 transgenic plants exhibit abnormal spike and floral phenotypes}

Among the $\mathrm{T}_{1}$ plants segregating for the three transgenic WAPO1 genes driven by their native promoters, the events showing significant differences in SNS also displayed spike abnormalities (Fig 4). One unusual phenotype was the presence of naked pistils at the base of spikelets 

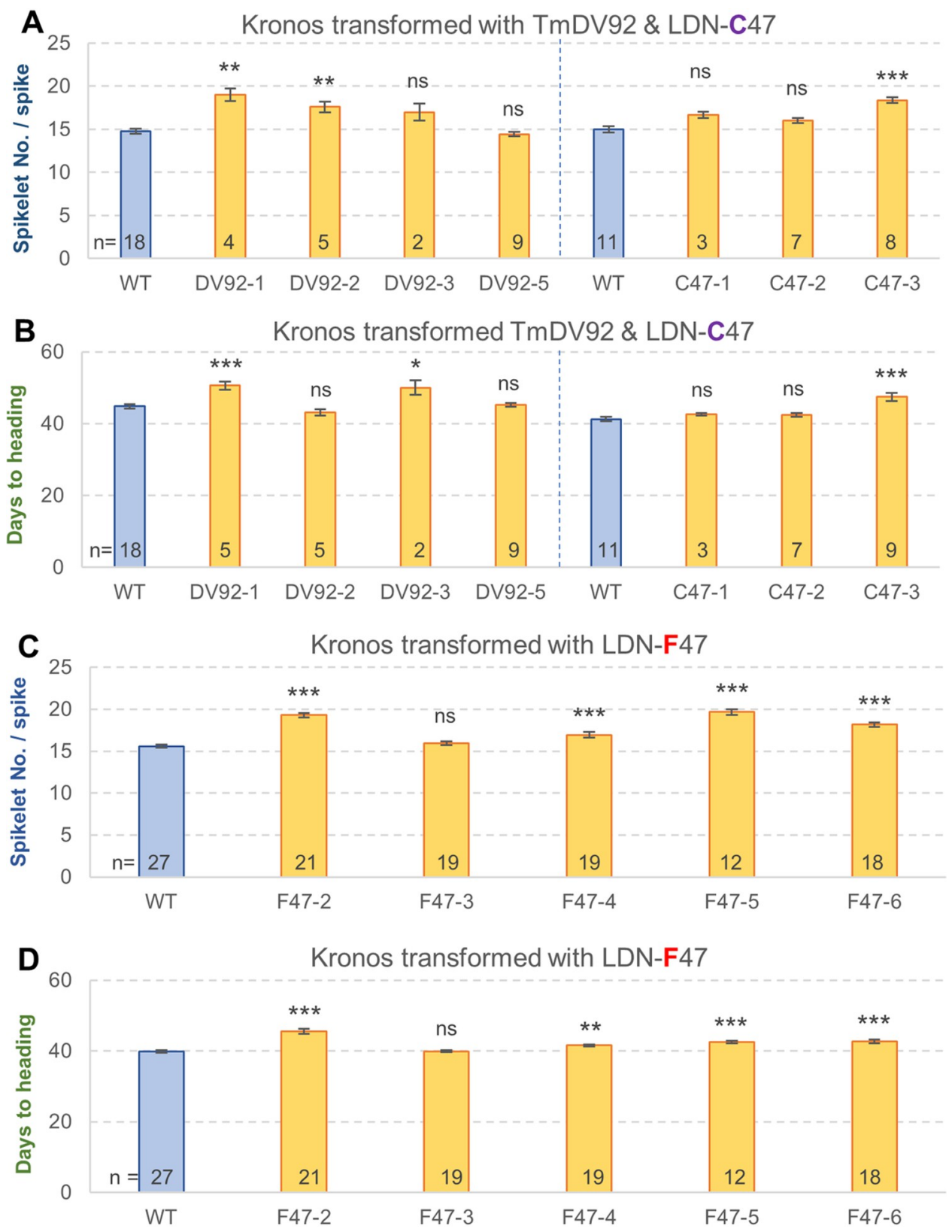

Fig 2. Effect of transgenic WAPO-A1 alleles on spikelet number per spike and heading date. (A-B) Greenhouse experiment comparing four independent TmDV92 transgenic lines and three independent lines carrying the LDN-C47 allele with their respective non-transgenic sister lines (pooled from different events). (C-D) Separate greenhouse experiment comparing five independent transgenic lines carrying the LDN-F47 allele with the pool of nontransgenic sister lines. (A and $\mathbf{C}$ ) Spikelet number per spike (only the spike from the main tiller was measured in each plant). (B and D) Heading date. Bars are averages and error bars are s.e.m. Numbers inside bars indicate number of plants and asterisks indicate $P$ values (Dunnett test) relative to the pool of non-transgenic sister lines carrying the same construct. ns $=$ not significant, ${ }^{*}=P<0.05,{ }^{* *}=P<0.01$ and $^{* * *}=P<0.001$. Data used for this figure and the corresponding statistical analyses are available in Data B in S1 Data.

https://doi.org/10.1371/journal.pgen.1009747.g002

located at basal positions of the spike (Fig 4A and 4B). This abnormality was observed in transgenic plants of events TmDV92-1 (in 4 out of $6 \mathrm{~T}_{1}$ ), LDN-C47-3 (in 3 out of $9 \mathrm{~T}_{1}$ ), LDN-F47-2 (in 8 out of $21 \mathrm{~T}_{1}$ ) and LDN-F47-6 (in 3 out of $18 \mathrm{~T}_{1}$ ) (Figs 4A, 4B and S2). Transgenic line 

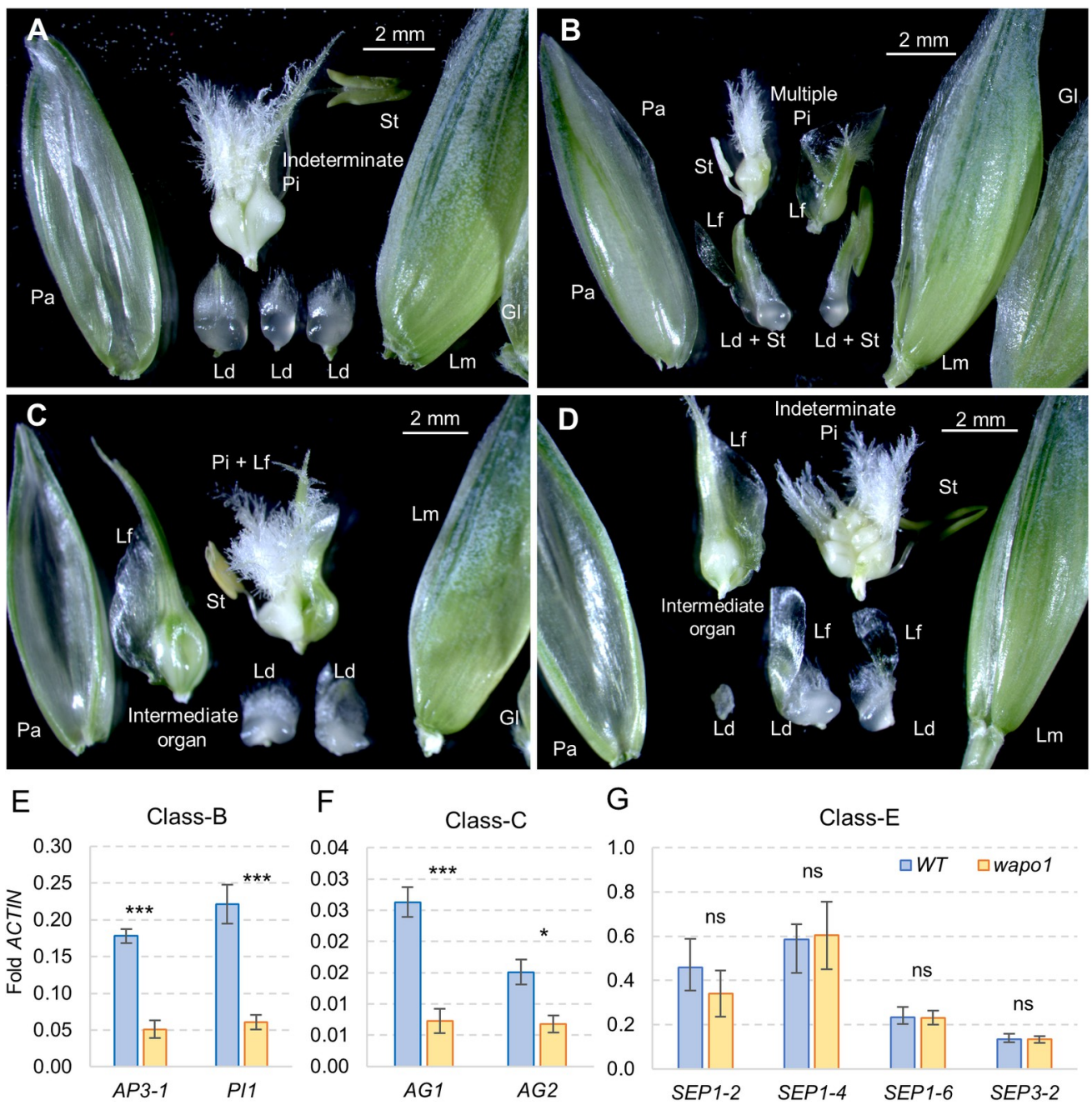

Fig 3. Floral abnormalities and expression profiles of Kronos wapo1 loss-of-function CRISPR mutant (wapo-A1 wapo-B1). (A) Mutant $\mathrm{T}_{1}-101$, floret one from basal spikelet showing three lodicules, one stamen and two fused pistils. (B) Mutant $\mathrm{T}_{1}-101,2^{\text {nd }}$ floret from $3^{\text {rd }}$ most distal spikelet. Lodicules fused with stamens and leafy tissue. Indeterminate pistils fused with leafy tissue. (C) Mutant $T_{1}-13,2^{\text {nd }}$ floret of basal spikelet. One lodicule fused with leafy tissue, and an intermediate organ fused with leafy tissue, and pistil fused with leafy tissue. (D) Mutant $\mathrm{T}_{1}-13,3^{\text {rd }}$ floret of basal spikelet. Lodicules with elongated leafy tissue, intermediate organ and indeterminate pistils. $\mathrm{Lm}=$ lemma, $\mathrm{Pa}=$ palea, $\mathrm{Ld}=$ lodicule, $\mathrm{St}=$ stamen, $\mathrm{Pi}=$ pistil, $\mathrm{Lf}=$ fused leafy tissue,$+=$ fused organs. Frequency of the different floral abnormalities is available in Data C in S1 Data. A wild-type Kronos floret is shown in Fig 4 as reference. (E-G) Expression analysis of class-A, -B, -C and -E MADS-box genes in Kronos and wapo1 mutants (MADS-box gene nomenclature is based on [20]). Primers for the qRT-PCRs are listed in S1 Table. Bars represent averages of four replicates and error bars are s.e.m. Each replicate is a pool of 12 developing spikes at the stamen primordia stage ( W4.0 in Waddington scale). $\mathrm{ns}=$ not significant, ${ }^{*}=P<0.05,{ }^{* *}=P<0.01$, and ${ }^{* * *}=P<0.001$. Data and statistical analyses used in E-G are available in Data D in S1 Data.

https://doi.org/10.1371/journal.pgen.1009747.g003

LDN-F47-2 had the most extreme phenotype showing multiple naked pistils, which were larger at the basal spikelets and transitioned to bracts in the more distal spikelets (S2A and S2B Fig).

A more frequent spike abnormality was the presence of smaller, densely packed spikelets in the distal region of the spike, ending in a small terminal spikelet. For simplicity, this spike abnormality is referred to hereafter as small terminal spikelet (Fig 4A red arrows and 4C). For event LDN-F47-3, which showed no significant differences in SNS, none of the $\mathrm{T}_{1}$ plants had 

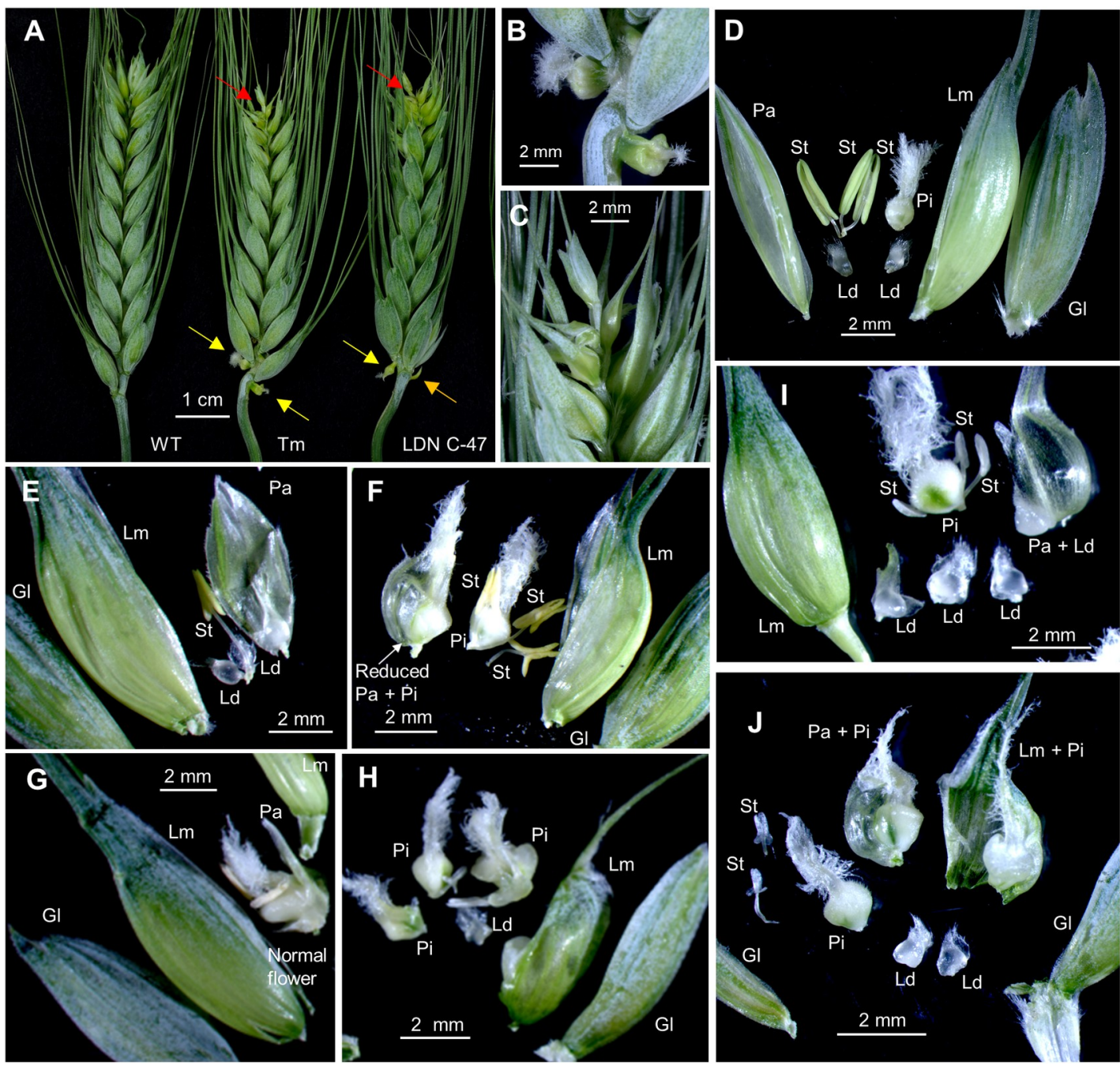

Fig 4. Floral abnormalities in Kronos plants transformed with genomic regions of WAPO-A1. (A) Spikes of non-transgenic control (WT) and transgenic lines TmDV92-1 and LDN-C47-3. (B) Detail of the naked pistils below the basal spikelets in TmDV92-1 (yellow arrows in A). (C) Detail of the abnormal distal end of the spike of TmDV92-1 (red arrows in A). (D) Normal floret from Kronos WT. (E and F) LDN-C47-3. (E) First floret in the $5^{\text {th }}$ spikelet from the base: reduced palea, one stamen and no pistil. (F) Second floret in $11^{\text {th }}$ spikelet from the base: no lodicules and extra pistils fused with small palea. ( $\mathbf{G}$ and $\left.\mathbf{H}\right)$ TmDV92-1. (G) First floret from basal spikelet: small palea and normal flower. (H) $17^{\text {th }}$ spikelet from the base: one lodicule, two stamens and indeterminate pistils. (I and J) LDN-F47-2. (I) Third floret from basal spikelet: four lodicules, one fused to palea. (J) Spikelet $19^{\text {th }}$ : two small stamens and three pistils (one fused to the palea and one to the lemma. $\mathrm{Lm}=$ lemma, $\mathrm{Pa}=$ palea, $\mathrm{Ld}=$ lodicule, $\mathrm{St}=$ stamen, $\mathrm{Pi}=$ pistil.

https://doi.org/10.1371/journal.pgen.1009747.g004

small terminal spikelets, whereas this phenotype was observed in $91.6 \%$ of the $T_{1}$ plants of LDN-F47-5, the event with the largest increase in SNS (26\%). The other three LDN-F47 events, showed significant but smaller increases in SNS (9 to 24\%) than the WT sister lines, and had small terminal spikelets in approximately half of the $\mathrm{T}_{1}$ lines (47 to $50 \%$ ).

Dissection of spikelets at different positions of the spike revealed increasing floral abnormalities towards the apical region. As reference, a normal floret from wild type Kronos is presented in Fig 4D. Examples of floral abnormalities are presented in Fig 4E and 4F (LDN-C473), Fig 4G and 4H (TmDV92-1), Fig 4I and 4J and S2 Fig (LDN-F47-2). The abnormal floral structures included (i) small paleae, (ii) variable numbers of lodicules, stamens and pistils per floret, and (iii) frequent fusions among these three organs and with lemmas and paleae 
(Fig 4E-4J). Transgenic line LDN-F47-2, which had the most extreme phenotype for naked pistils (S2A and S2B Fig), also showed multiple floral defects. Spikelets near the terminal spikelet had higher numbers of pistils and reduced numbers of other organs (S2C Fig), whereas basal spikelets showed larger glumes, lemmas and stamens, particularly in the distal florets (S2D-S2G Fig).

For all three transgenic lines, the progeny of plants carrying the transgenes showed significantly higher $(P<0.001)$ transcript levels at W3.5 than their sister lines without the transgene (all plants have the endogenous $\mathrm{H} 1$ WAPO-A1 allele) (Fig 5A-5C). In all three experiments, the progeny of plants from events with strong spike phenotypes (naked pistils and small terminal spikelet) showed higher WAPO-A1 transcript levels (Fig 5A-5C), and stronger reductions in number of florets per spikelet (Fig 5D-5F) and average spikelet fertility (Fig 5G-5I) than the progeny of the events with weaker spike phenotypes and intermediate WAPO-A1 expression levels. The progeny of F47-2-1 segregated for weak and strong spike phenotypes and the
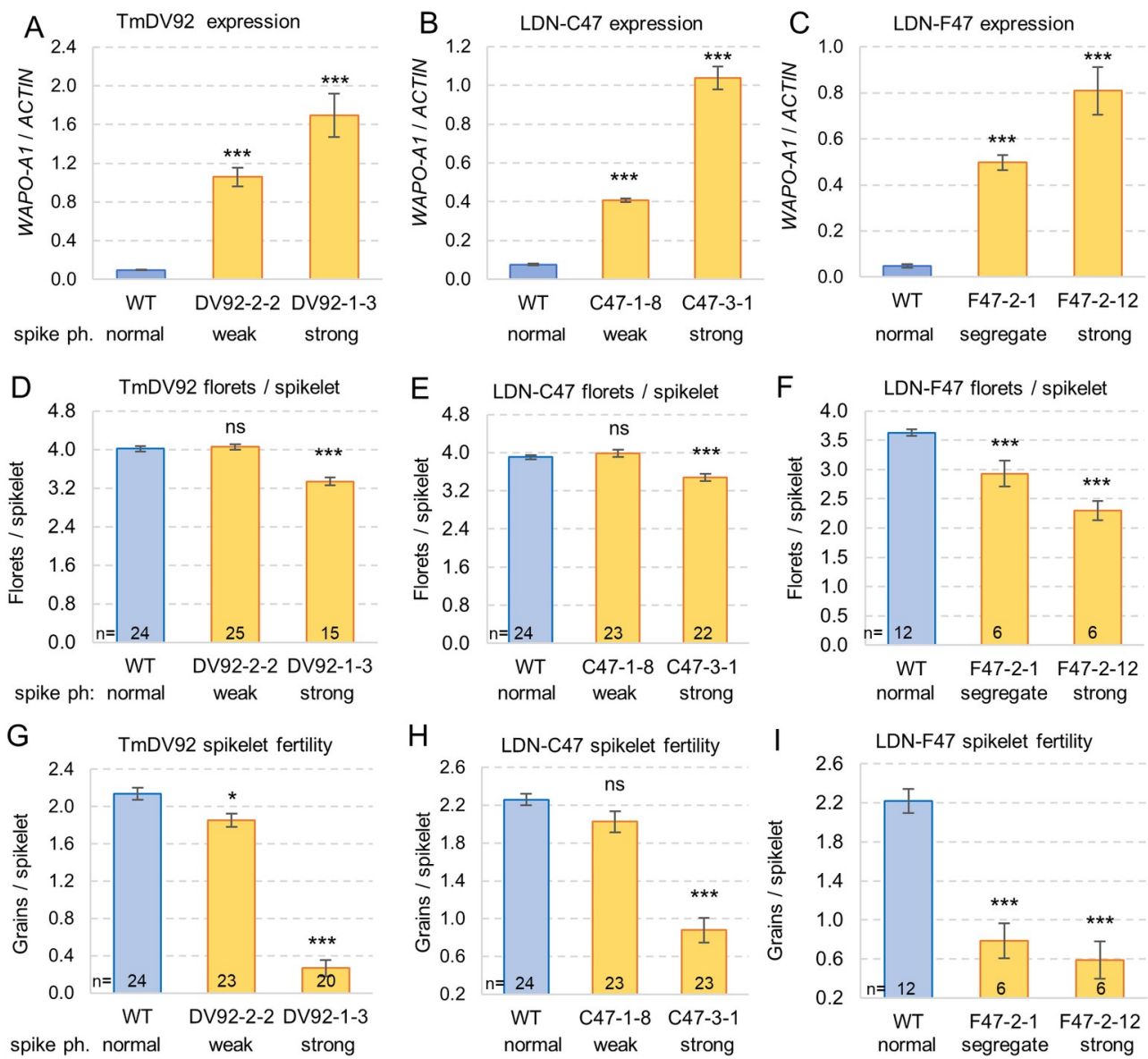

Fig 5. WAPO-A1 transcript levels and fertility in Kronos transgenic plants. (A-C) qRT-PCR transcript levels calculated with the $\triangle \mathrm{Ct}$ method using ACTIN as an endogenous control. Bars are averages of four replications and each replication corresponds to a pool of 6-9 developing spikes at the floret primordia stage (W3.5). Spike ph. $=$ spike phenotype, weak = differences in SNS, strong = naked pistils and small terminal spikelet, segregate $=$ segregation for strong and weak spike phenotypes (F47-2-1). (D-F) Average floret number per spikelet (G-I) Spikelet fertility calculated as GNS / SNS. Only one spike per plant was measured. Kronos plants transformed with (A, D and G) TmDV92 (from T. monococcum). (B, E and H) LDN-C47, and (C, F and I) LDN-F47. The first number in a transgenic plant designation corresponds to the transformation event. ns $=$ not significant, ${ }^{*}=P<0.05,{ }^{* * *}=P<0.001$, indicate Dunnett test $P$ value relative to the WT sister line. Bars represent averages and error bars are s.e.m. Numbers at the base of the bars indicate plants analyzed. Data and statistical analyses are available in Data E in S1 Data.

https://doi.org/10.1371/journal.pgen.1009747.g005 
number of florets per spikelet and spikelet fertility was more affected than in the progenies of the other two constructs with intermediate levels of WAPO-A1 expression. In all three constructs, the reductions in spikelet fertility were stronger than the reductions in the number of florets per spikelet, suggesting that both reduced floret fertility (due to floral defects) and reduced number of florets per spikelet (due to smaller distal spikelets) contributed to the drastic reductions in spikelet fertility and GNS in the transgenic plants with high WAPO-A1 expression.

\section{WAPO1 is expressed in inflorescence, spikelet and floral meristems}

To better characterize the expression profile of WAPO1, we investigated its spatial and temporal distribution during spike and spikelet development in Kronos (Fig 6) and diploid T. monococcum (S3 Fig) by in situ hybridization. In both species, weak expression of WAPO1 was detected in the inflorescence meristem (IM) at the double ridge stage (W2.5, Figs 6A and S3A) and in both the IM and spikelet meristems (SM) at the subsequent W3.0 stage (Figs 6B and $\mathrm{S} 3 \mathrm{~B})$. At W3.25 -W3.5, a strong expression of WAPO1 was detected in the incipient floret meristems (Figs 6C and S3C). In summary, WAPO1 expression in the inflorescence and spikelet meristems correlates well with its effects on the timing of terminal spikelet induction, whereas its expression in the floret meristem correlates with its role in floral development and the flower defects observed in WAPO1 mutants and transgenic plants.

\section{Natural variation in $W A P O-A 1$ is associated with changes in SNS}

In addition to the validation of WAPO-A1 as the causal gene for the 7AL SNS QTL, we investigated the effect of the different WAPO-A1 alleles on SNS and GNS. In our previous study, we

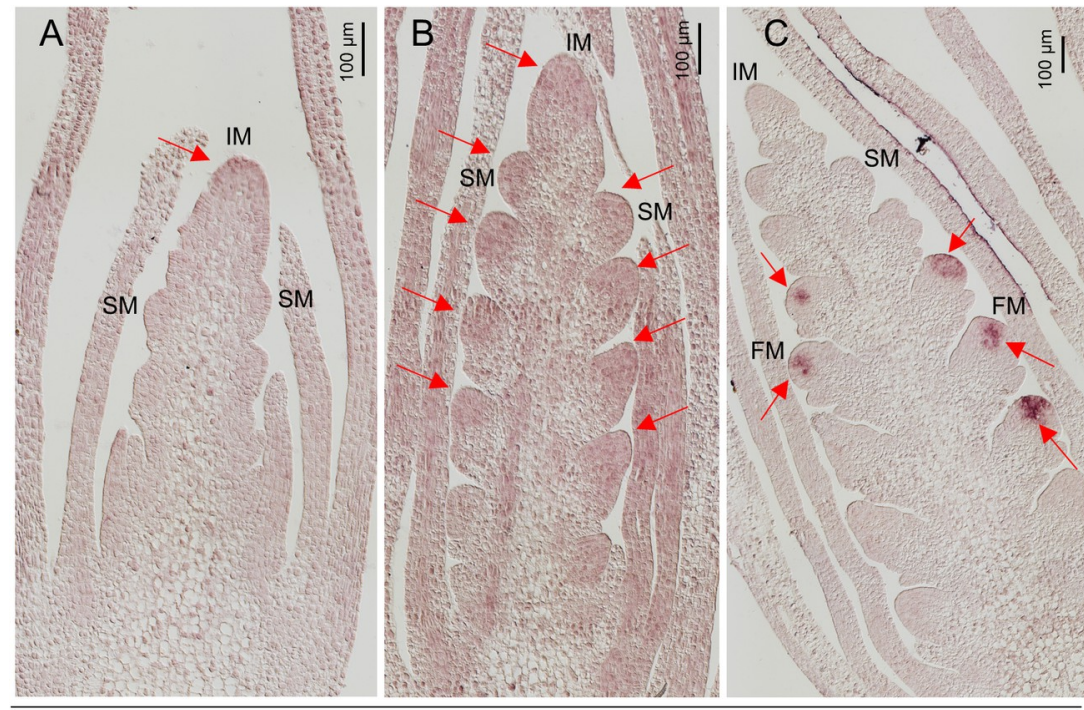

WAPO1 antisense

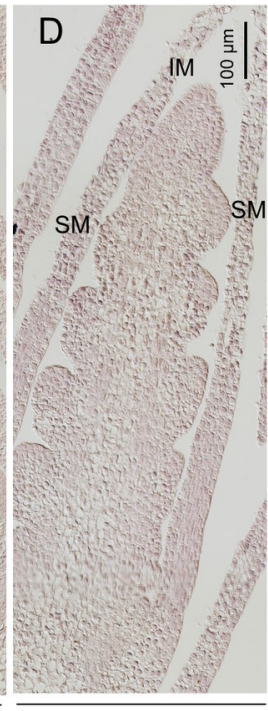

WAP01 sense

Fig 6. In situ hybridization analysis of WAPO-A1 in sections of developing spikes of tetraploid wheat Kronos. (A-C) WAPO1 antisense probe. (D) WAPO1 sense probe used as a negative control in a developing spike at W2.5 with undifferentiated spikelet meristems (SM). (A) Developing spike at the double ridge stage (W2.5). (B) Early spike development with undifferentiated spikelet meristems (W3.0). Weak WAPO1 expression was detected in the inflorescence meristem and spikelet meristem. (C) Spikes at W3.25-W3.5 showing strong expression of WAPO1 in the incipient floret meristems of the more developed spikelets closer to the center of the spike. W values indicate developmental stages based on the Waddington scale. IM = inflorescence meristem, SM = spikelet meristem, and $\mathrm{FM}=$ floret meristem. Red arrows indicate $W A P O-A 1$ hybridization signals. 
A

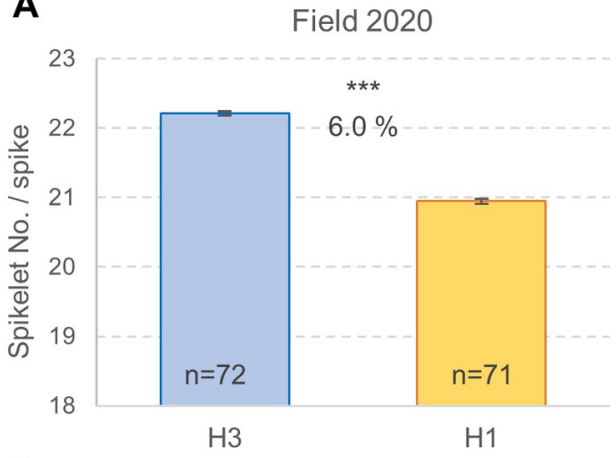

C
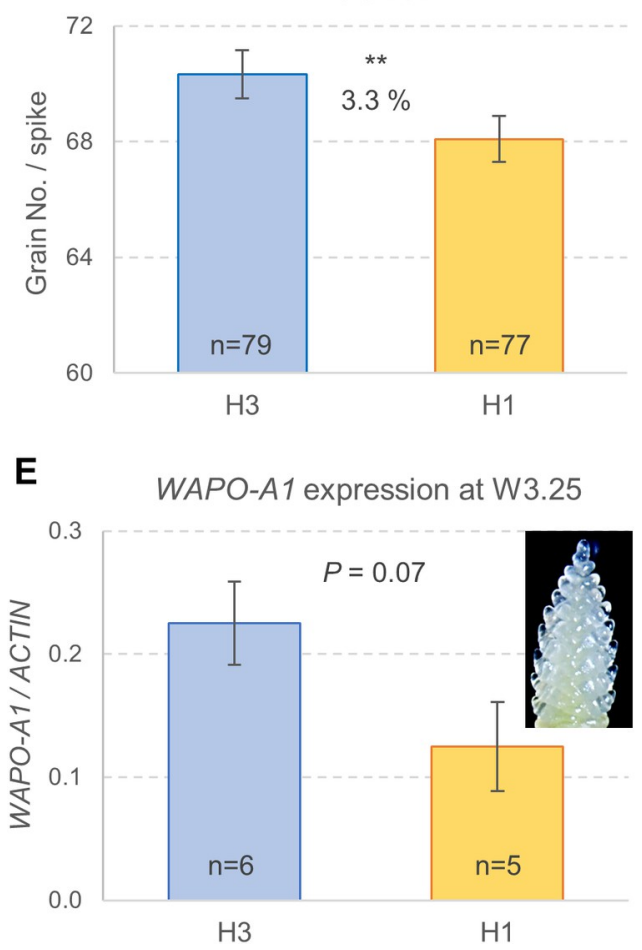

B

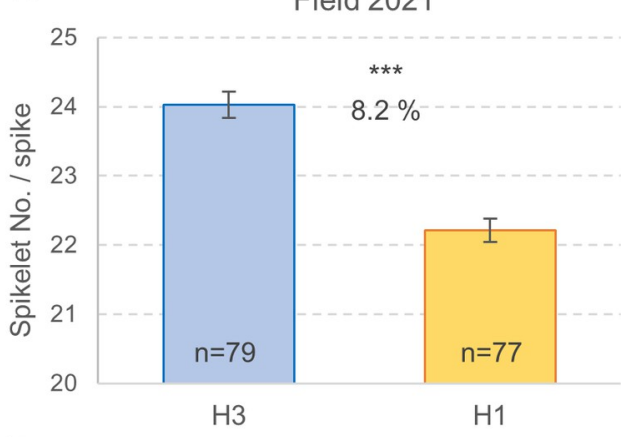

D

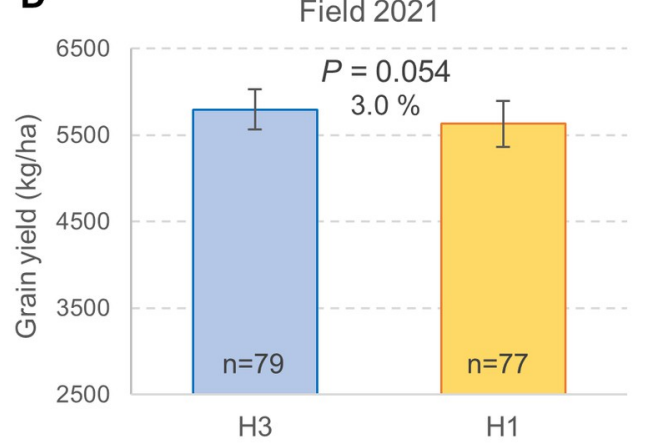

$\mathbf{F}$

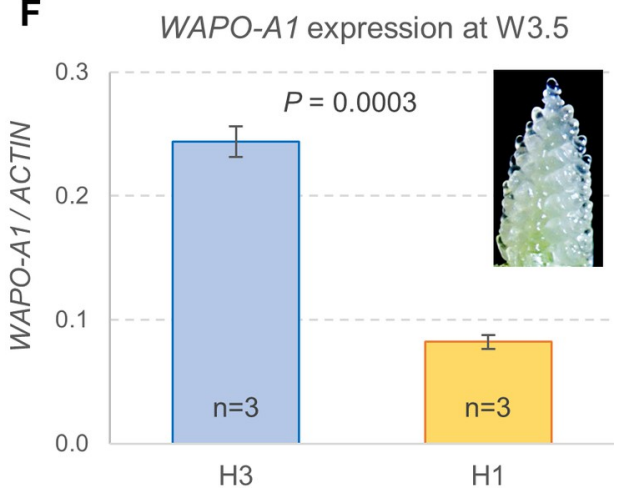

Fig 7. Effect of WAPO-A1 haplotypes $\mathrm{H} 1$ and $\mathrm{H} 3$ on SNS and WAPO-A1 transcript levels. (A) $2020 \mathrm{RCBD}$-split plot field experiment. (B-D) 2021 RCBD split-plot field experiment. (B) Spikelet number per spike. (C) Grain number per spike. (D) Grain yield (kg/ha) (E-F) Independent qRT-PCR experiments comparing H1 and H3 transcript levels of WAPO-A1 relative to ACTIN using the $2^{\Delta \mathrm{Ct}}$ method. (E-F) Pools of 3-8 developing spikes from the main tiller. (E) Collected when lemma primordia were present (W3.25). (F) Collected when floret primordia were present (W3.5). Bars represent averages and error bars are s.e.m. Numbers inside the bars indicate the number of replications. ns $=$ not significant, ${ }^{*}=P<0.05,{ }^{* *}=P<0.01$ and $^{* * *}=P<0.001$. Data used for this figure are available in Data G in S1 Data, and the statistical analyses in S3 Table.

https://doi.org/10.1371/journal.pgen.1009747.g007

established that the WAPO-A1 allele in the $\mathrm{H} 2$ haplotype was associated with higher SNS than the $\mathrm{H} 1$ and $\mathrm{H} 3$ haplotypes [10], but we did not determine the relative effects of the $\mathrm{H} 1$ and $\mathrm{H} 3$ haplotypes on SNS. To address this question, we performed two field experiments in 2020 (Fig 7A and panel A in S3 Table) and 2021 (Fig 7B-7D and panels B-G in S3 Table), both organized in an RCBD-split plot design. Both experiments had 10 replications, each including eight $\mathrm{F}_{4: 6}$ heterozygous inbred families (HIF) as main plots and homozygous $\mathrm{H} 1$ and $\mathrm{H} 3$ sister lines as subplots. Average SNS for plants homozygous for the $\mathrm{H} 3$ haplotype was 6\% higher than for those homozygous for the $\mathrm{H} 1$ haplotype in the 2020 experiment (1.3 spikelets) and $8.2 \%$ higher 
in the 2021 experiment (1.8 spikelets). The overall differences in SNS were highly significant both years $(P<0.0001$, Fig $7 \mathrm{~A}$ and $7 \mathrm{~B}$, panels $\mathrm{A}$ and B in $\mathrm{S} 3$ Table). Within individual families, differences in SNS between $\mathrm{H} 1$ and $\mathrm{H} 3$ were significant for six out of the 8 families in 2020 and for all families in 2021 (panels A and B S3 Table).

In the 2021 experiment, we had sufficient grains to use small plots $\left(1.86 \mathrm{~m}^{2}\right)$ as experimental units and to provide a preliminary estimate of grain yield. In this experiment, the $\mathrm{H} 3$ haplotype showed a $3.3 \%$ increase in GNS ( $P=0.0085$, Fig $7 \mathrm{C}$ and panel C in S3 Table). The increase in GNS was smaller than the increase in SNS $(8.2 \%)$ because it was partially offset by a $4.6 \%$ decrease in fertility (grains per spikelet) in $\mathrm{H} 3$ relative to $\mathrm{H} 1(P<0.001$, panel $\mathrm{D}$ in S3 Table and Data F in S1 Data). The H3 haplotype was also associated with a 1.6\% decrease in kernel weight $(P=0.0281$, panel $\mathrm{E}$ in S3Table) relative to $\mathrm{H} 1$. The balance of these opposite effects was a $3.0 \%$ increase in grain yield, but the difference was marginally not significant $(P=0.0545$, Fig 7D and panel F in S3 Table). In this experiment, we did not detect significant differences in heading time ( $P=0.5987$, panel $\mathrm{G}$ in S3 Table).

To test if the differences in SNS between $\mathrm{H} 1$ and $\mathrm{H} 3$ haplotypes were associated with differences in the expression levels of WAPO-A1, we performed three independent qRT-PCR experiments comparing homozygous $\mathrm{H} 1$ and $\mathrm{H} 3$ sister lines derived from $\mathrm{F}_{4: 6} \mathrm{HIF} \# 120$.

Developing spikes from the main tiller were collected at the lemma primordia stage (W3.25) for the first two experiments and at the floret primordia stage (W3.5) for the third experiment (Fig 7E and 7F). The first two experiments at W3.25, which were analyzed together using experiment as block, showed 1.8-fold higher transcript levels in $\mathrm{H} 3$ than in H1, but the difference was marginally not significant $(P=0.07$, Fig $7 \mathrm{E}$ and Data G in S1 Data). In the third experiment at W3.5, transcript levels in $\mathrm{H} 3$ were 3.0-fold higher than in $\mathrm{H} 1$ and the differences were highly significant $(P=0.0003$, Fig $7 \mathrm{~F}$ and Data G in S1 Data).

In the same field used for comparing the $\mathrm{H} 1$ and $\mathrm{H} 3$ haplotypes in 2021, we also compared the relative effects of the $\mathrm{H} 2$ and $\mathrm{H} 1$ haplotypes in near isogenic lines (NILs) of tetraploid wheat Kronos and hexaploid wheat GID4314513 (a high-biomass line from CIMMYT). In the Kronos $\mathrm{BC}_{3} \mathrm{~F}_{3}$ NILs, the H2-NILs showed $17.7 \%$ more spikelets per spike $(P<0.0001$, Fig $8 \mathrm{~B})$ and $6.7 \%$ more grains per spike ( $P=0.025$, Fig 8 C) than the H1-NILs (panels A-B in S4 Table). The lower percent increase in GNS than in SNS can be partially explained by a $9.2 \%$ reduction in spikelet fertility (GNS/SNS) in the H2-NILs $(P<0.0001$, Fig 8C and panel C in S4 Table). Grain weight per spike (grain number $\mathrm{x}$ grain weight) was $7.1 \%$ higher in the H2-NILs than in the H1-NILs, but the differences were not significant $(P=0.11$, Fig 8D and panel D in S4 Table). The H2-NILs were also associated with a non-significant increase in grain weight $(0.4 \%)$ and a non-significant delay in heading time ( $0.7 \%$ panels E-F in S4 Table). Total grain yield was not estimated in this experiment because experimental units were single rows.

In the hexaploid $\mathrm{BC}_{5} \mathrm{~F}_{3}$ NILs, tested in small plots, we detected a $6.1 \%$ increase in SNS in the H2-NILs relative to the H1-NILs $(P=0.0092$, Fig $8 \mathrm{E}$ and panel G in S4 Table) and an 8.4\% increase in grain yield that was marginally significant $(P=0.0497$, Fig $8 \mathrm{~F}$ and panel $\mathrm{H}$ in $\mathrm{S} 4$ Table). The $\mathrm{H} 2$ haplotype was also associated with non-significant differences relative to $\mathrm{H} 1$ for GNS $(+2.7 \%)$, fertility $(-3.3 \%)$, kernel weight $(+1.8 \%)$ and grain weight per spike $(+4.4 \%$, panels I-L in S4 Table).

\section{Discussion}

In our previous study, we identified WAPO-A1 as the leading candidate gene for the 7AL SNS QTL based on two high resolution genetic maps [10]. In this study, we demonstrate that WAPO1 is both necessary and sufficient to increase SNS in wheat and, therefore, that $W A P O-A 1$ is the causal gene underpinning the 7AL QTL for SNS. 
A

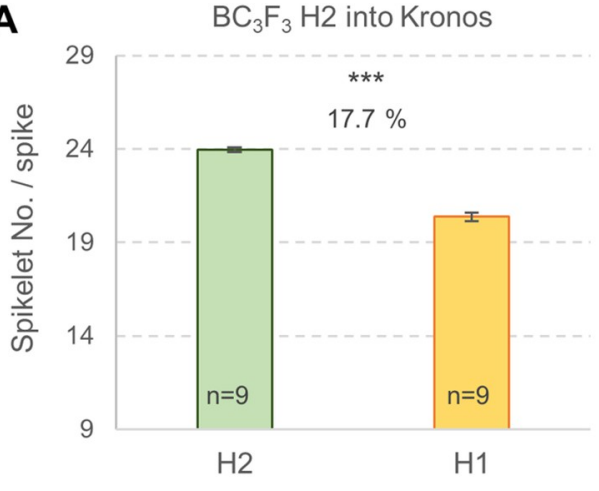

C

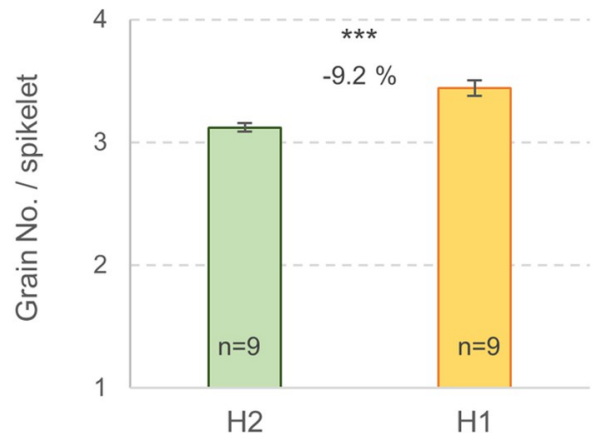

E

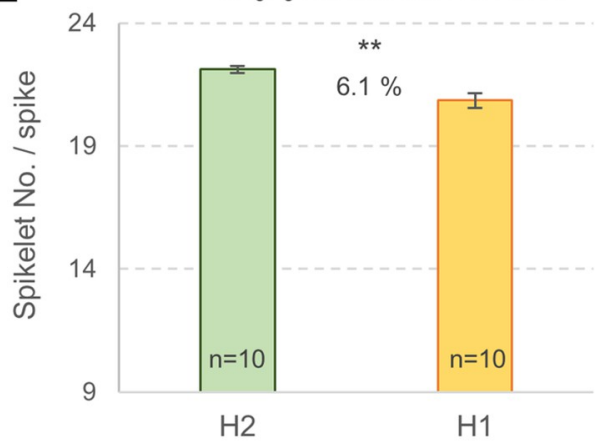

B

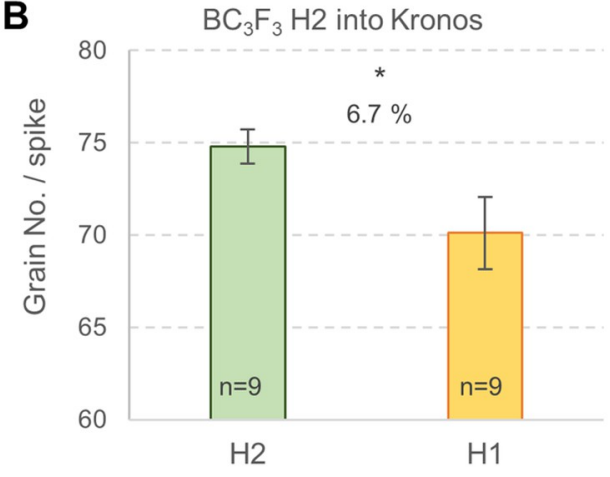

D

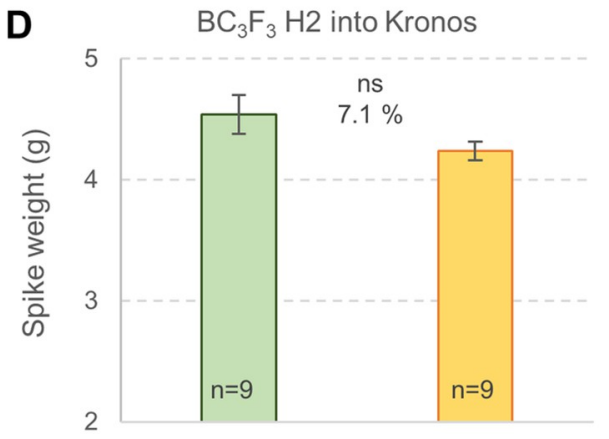

$\mathrm{H} 2$

$\mathrm{H} 1$

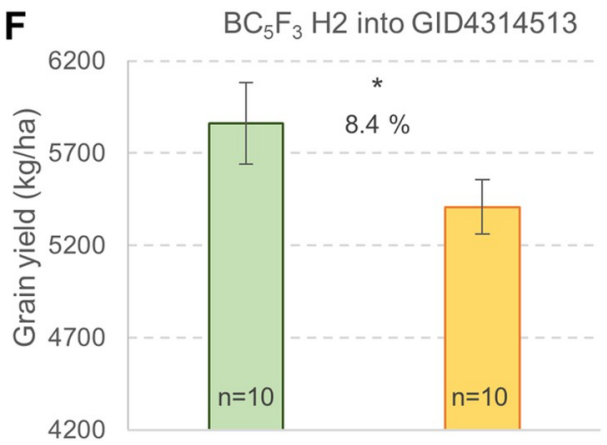

$\mathrm{H} 2$

$\mathrm{H} 1$

Fig 8. Effect of WAPO-A1 haplotype $\mathrm{H} 2$ introgressed into tetraploid Kronos and the high-biomass hexaploid line GID4314513 (both H1). Experiments performed in the field in 2021. (A-D) Homozygous $\mathrm{BC}_{3} \mathrm{~F}_{3}$ sister lines (CRD, $\mathrm{n}=9,1$-m rows, 10 spikes measured per row). (A) Spikelet number per spike. (B) Grain number per spike. (C) Grain number per spikelet (spikelet fertility). (D) Grain weight per spike. (E-F) Homozygous $\mathrm{BC}_{5} \mathrm{~F}_{3}$ sister lines (RCBD, $\mathrm{n}=10,1.86 \mathrm{~m}^{2}$ plots, 4 spikes measured per plot). Error bars are s.e.m. $\mathrm{ns}=$ not significant, ${ }^{*}=P<0.05,{ }^{* *}=P<0.01$, ${ }^{* * *}=P<0.001$. Data used to generate this figure are available in Data H in S1 Data and the statistical analyses in S4 Table.

https://doi.org/10.1371/journal.pgen.1009747.g008

\section{Potential mechanisms involved in WAPO1 effect on SNS}

Differences in WAPO-A1 transcript levels in different genotypes correlate well with differences in SNS. The reduced expression of the WAPO-A1 allele (115-bp promoter deletion) in $\mathrm{H} 1$ relative to the $\mathrm{H} 2$ [10] and $\mathrm{H} 3$ haplotypes (Fig 7) is associated with lower SNS in $\mathrm{H} 1$ relative to the other two haplotypes. Similarly, the higher transcript levels of WAPO-B1 relative to WAPO-A1 during spike development (S1 Fig) is associated with stronger effects of the wapo-B1 mutation on SNS relative to the wapo-A1 mutation (Fig 1D). These results, together with the partial 
codominant effect of WAPO1 on SNS (Fig 1E) and the increased SNS in transgenic plants with additional genomic copies of WAPO-A1 (Fig 2), support the hypothesis that higher WAPO1 transcript levels can drive increases in SNS.

Increases in SNS are associated with a delay in the transition of the inflorescence meristem (IM) into a terminal spikelet, and our in situ hybridization results confirmed that WAPO1 is weakly expressed in the IM at the double ridge stage in both diploid (S3A Fig) and tetraploid wheat (Fig 6A). During the early stages of spikelet development, we detected hybridization signals in the IM and the spikelet meristems (SM, Fig 6B). As spike development approached the formation of the terminal spikelet and the lateral spikelets started to differentiate, the WAPO1 hybridization signal was no longer detected in the IM and was replaced by a strong signal in the early floret meristems (FM, Fig 6C).

The barley ortholog of WAPO1, HVAPO1, has been also detected in the IM and FM of the developing barley spikes by in situ hybridization [21]. Those experiments showed a strong HvAPO1 hybridization signal in both the IM and FM at the time of spikelet differentiation (see Fig S10 in reference [21]). By contrast, at the time when WAPO1 was highly expressed in the FM in the wheat developing spike (W3.25-W3.5), we failed to detect WAPO1 in the IM (Figs $6 \mathrm{C}$ and $\mathrm{S} 3 \mathrm{C}$ ). It would be interesting to investigate if the stronger and extended expression of HvAPO1 in the barley IM during floret development contributes to the different fate of the IM in barley (indeterminate inflorescence) compared to wheat (determinate inflorescence).

In rice, $A P O 1$ is also expressed in the IM and in the primary and secondary branch meristems of the panicle, and apo1 mutants have a reduced number of branches and spikelets [11]. In rice, it was shown that APO1 physically interacts with LFY (APO2), and that the two genes act cooperatively to control spikelet number per panicle [15]. Similar in vitro and in vivo interactions have been reported between the Arabidopsis homologs UFO and LFY [14,22]. Mutants for both genes show similar defects in inflorescence structure and morphology, further suggesting that UFO and LFY act cooperatively to promote floral-meristem identity [23]. Based on the rice and Arabidopsis results, we hypothesize that $L F Y$ may participate in the mechanism by which WAPO1 regulates SNS in wheat. In Arabidopsis, $L F Y$ has been shown to act both as a transcriptional activator of the SQUAMOSA MADS-box gene AP1 [24] and as a repressor of the SVP MADS-box genes AGL24 and SVP [25]. Therefore, it would be interesting to investigate the roles of WAPO1 and LFY in the regulation of the wheat AP1 homologs (VRN1, FUL2 and FUL3) and $S V P$ homologs (VRT2 and $S V P 1$ ), which have been previously shown to be involved in the regulation of SNS in wheat [26,27].

\section{Floral and spike abnormalities in mutant and transgenic WAPO-A1 plants}

In addition to its role in the activation of $A P 1$, LFY has been shown to bind to regulatory regions of APETALA3 (AP3, a class-B floral gene) and AGAMOUS ( $A G$, a class-C floral genes) in Arabidopsis [14,28-30]. The activation of $A P 3$ expression requires the activity of both $L F Y$ and $U F O$, which likely explains the reported downregulation of class-B genes in the $u f o$ mutant in Arabidopsis [13,14] and class- $C$ genes in the apo 1 mutant in rice [12]. In the developing spikes of the wheat wapo1 null mutants, we also observed downregulation of class-B and -C but not class-E genes relative to the WT, suggesting a conserved molecular mechanism.

Class-B and -C MADS-box genes in Arabidopsis are associated with petal, stamen and pistil identity, which explains the stronger abnormalities detected in the ufo mutants in these three inner floral whorls. It has been shown that ectopic expression of both PI and AP3 rescues the floral organ identity defects of the Arabidopsis ufo mutant [31]. Stronger floral defects in the inner whorls of the flowers than in glumes and lemmas have been observed before in the rice apo1 mutant [12], and in this study in the wheat wapo1 mutant. The related floral defects 
observed in the $u f o$, apo1 and wapo1 mutants suggest functional conservation of these homologous genes across the monocot eudicot divide.

The strong expression of WAPO1 in the floret meristem (Figs 6C and S3C), supports the critical role of this gene in wheat floral development. Transgenic wheat plants with additional WAPO1 genomic copies shared some floral aberrations with the wapo1 mutant, including differences in the number of lodicules, stamens and pistils and fusion among organs. In addition, mutants and transgenic plants with strong floral abnormalities showed reduced fertility (Fig $5 \mathrm{G}$ and 5I). These results suggest that decreases or increases in WAPO1 expression can lead to abnormal development in the three inner whorls of the floret. Transgenic WAPO-A1 plants showed additional phenotypes not observed in the wapo1 mutant, including reduced and sometimes curved paleae (homologous to sepals), a compact spike tip with a small terminal spikelet, and a significant delay in heading time.

A less frequent but unusual phenotype in the transgenic WAPO1 plants was the presence of naked pistils. A similar phenotype has been described in the Arabidopsis ufo mutants, where structures resembling normal pistils were formed at the end of primary and coflorescence shoots $[13,32]$. The naked pistils in the transgenic wheat plants appeared adjacent to and directly below normal spikelets, in a position similar to the paired spikelets observed in loss-of-function mutants for $p p d-D 1$ and $f t-B 1$ in hexaploid wheat [33]. However, the paired spikelets in the previous two mutants were more frequent in the central part of the spike, whereas in the WAPO-Al transgenic plants the naked pistils were more frequent in the basal region of the spike. Based on the terminal position of the naked pistils in Arabidopsis ufo mutants, we hypothesize that the naked pistils in the transgenic wheat plants may be located at the end of a short branch including one lateral spikelet. Branches in the lower nodes of the wheat spike have been reported in the Branched head mutants, which carry mutations in an AP2/ERF transcription factor [34,35].

\section{Effects of different $W A P O-A 1$ natural alleles and their potential applications in wheat breeding}

Our previous study showed that the $\mathrm{H} 2$ haplotype was associated with larger increases in SNS than the $\mathrm{H} 1$ and $\mathrm{H} 3$ haplotypes in several segregating populations [10]. The favorable effect of $\mathrm{H} 2$ on SNS was observed in spring and winter wheats and in different wheat market classes, but these increases did not always result in higher grain yield. In genotypes or environments with insufficient resources to develop and/or fill the extra grains, increases in SNS were partially or completely offset by decreases in fertility and/or grain weight. However, when $\mathrm{H} 2$ was present in well adapted and productive genotypes, and the plants were grown in favorable environments, the increases in SNS were associated with significant increases in grain yield [10]. In this study, we also observed significant $8.4 \%$ increases in total grain yield when the $\mathrm{H} 2$ haplotype was introgressed into a highly productive hexaploid line from the high-biomass program from CIMMYT and the plants were grown under favorable irrigation and fertilization conditions. This is a promising preliminary result, but needs to be further validated with larger yield trials in different genetic backgrounds.

The positive effects of the $\mathrm{H} 2$ haplotype on SNS in the less productive tetraploid wheat Kronos was partially offset by a reduction in spike fertility $(-9.2 \%)$, which resulted in a smaller increase in GNS (6.7\%) than in SNS (17.7\%). In this experiment we did not observe a negative effect of the $\mathrm{H} 2$ haplotype in grain weight. The balance of these different effects was a positive $7.1 \%$ increase in grain yield per spike, but this difference was not significant $(P=0.1078)$. Based on these results, we hypothesize that when the plant has more available spikelets, it adjusts the number of grains or their filling to the available resources, depending on the timing during development when the plant faces the limited resources. 
An increase in the frequency of the $\mathrm{H} 2$ haplotype was previously described in common wheat, from less than $50 \%$ in old landraces to more than $80 \%$ in modern wheat varieties [10]. This suggests that selection for high SNS, GNS, or grain yield may have accelerated the increases in $\mathrm{H} 2$ frequencies. By contrast, in tetraploid wheat the $\mathrm{H} 1$ haplotype replaced the $\mathrm{H} 3$ haplotype and became almost fixed in modern durum varieties (99\%) [10]. This increase in H1 frequency cannot be explained by selection for higher SNS or GNS, because our field experiment showed that $\mathrm{H} 1$ has significantly lower SNS and GNS than H3 (Fig 7). Since durum wheat has much larger grains than common wheat, we speculate that selection for larger grains may have resulted in an indirect selection for reduced grain number, favoring the $\mathrm{H} 1$ allele. This indirect selection was likely driven by the negative correlation between grain number and weight, a trade off that is magnified in source-limited varieties and/or suboptimal environments.

A similar result has been recently reported for the FT-A2 gene, where the derived A10 allele associated with increases in SNS was found at high frequency in common wheat ( 60\%) but was almost absent in modern durum wheat commercial varieties (0.7\%) [36]. These results suggest that other genes involved in the regulation of spike development may have differential frequencies between common and durum wheat as a result of this putative selection for large grains.

However, it is also possible that the derived WAPO-A1 $\mathrm{H} 2$ and FT-A2 A10 alleles were never properly tested in modern durum wheat varieties. The WAPO-A1 $\mathrm{H} 2$ haplotype was found in only one accession from Syria in a survey of 364 durum wheat varieties [10]. Similarly, the FT-A2 A10 allele was found only in three accessions from Oman and Turkey in a survey of 417 durum wheat varieties [36]. In this study, we show that the transfer of the WAPO-A1 $\mathrm{H} 2$ haplotype from common wheat into the tetraploid wheat Kronos was associated with significant increases in SNS and GNS. A similar favorable effect of $\mathrm{H} 2$ relative to $\mathrm{H} 1$ on SNS was previously reported in a segregating population generated from a cross between cultivated emmer and durum wheat in field trials in Fargo (ND) [10]. These are promising results, but they still require validation in different durum varieties and larger yield trials. To facilitate the introgression of the $\mathrm{H} 2$ haplotype in durum wheat, we deposited the Kronos NIL with the H2 introgression in the National Small Grains Collection (PI 698810).

In summary, the validation of WAPO-A1 as the causal gene of the 7AL SNS QTL and of the positive effects of the $\mathrm{H} 2$ haplotype on spikelet and grain number per spike, provides wheat breeders a new tool to improve sink traits with limited effects on heading time. We hypothesize that the introgression of the $\mathrm{H} 2$ haplotype into varieties that are not limited in source traits (e.g. varieties with high biomass) can result in increases in total grain yield in favorable environments.

\section{Materials and methods}

\section{Ethyl methanesulfonate (EMS) induced mutations in WAPO-A1}

We screened a database of sequenced EMS induced mutations in the tetraploid wheat variety Kronos [16] using the sequence of WAPO-A1 (TraesCS7A02G481600). Kronos has the $W A P O-A 1 \mathrm{H} 1$ haplotype, which carries a 115-bp deletion in the promoter and a change from aspartic acid to asparagine at position $384(\mathrm{D} 384 \mathrm{~N})$. For the A-genome homeolog (WAPO-A1), we identified line K4222 carrying a mutation that results in the replacement of a tryptophan by a premature stop codon at position 216 of the protein $\left(\mathrm{W} 216^{*}\right)$. For the Bgenome homeolog (WAPO-B1), we identified five mutations that resulted in amino acid changes but none generated premature stop codons or altered splicing sites; hence, our first studies of the EMS mutants only included K4222. We crossed the K4222 mutant to the nonmutagenized Kronos, self-pollinated the $\mathrm{F}_{1}$, and selected sister lines homozygous for the 
mutant (wapo-A1) and the WT alleles (Wapo-A1) from the segregating $\mathrm{F}_{2}$ plants. The derived $\mathrm{F}_{3}$ plants were evaluated in the greenhouse and the $\mathrm{F}_{4}$ plants in the field. To further reduce background mutations, we generated $\mathrm{BC}_{1} \mathrm{~F}_{2}$ homozygous sister lines by backcrossing the $\mathrm{F}_{1}$ with Kronos, self-pollinating the $\mathrm{BC}_{1}$, and selecting homozygous plants in the next generation. The derived $\mathrm{BC}_{1} \mathrm{~F}_{3}$ grains were planted in a field experiment.

For the greenhouse experiment, we used two $\mathrm{F}_{3}$ plants per pot $(3.8 \mathrm{~L})$ and measured average SNS per plant. In the first field experiment, we planted homozygous mutant and WT $\mathrm{F}_{4}$ sister lines in a complete randomized design (CRD) with 10 replicates per genotype. Each replicate included a row of 2-5 plants spaced 0.3 meters apart. Average SNS per row was calculated by measuring SNS from 3 spikes per plant. In the second field experiment, we planted $\mathrm{BC}_{1} \mathrm{~F}_{3}$ plants $0.3 \mathrm{~m}$ apart segregating for the WAPO-A1 alleles. Genotyping of these plants revealed 11 homozygous wapo- $A 1$ and 8 homozygous $\mathrm{WT}$ within the same $\mathrm{BC}_{1}$ family $\# 54$. For each plant, we determined the average SNS from four spikes. Both field experiments were conducted at the University of California Experimental Field Station in Davis, referred to hereafter as UC Davis, during the months of October 2019 and June 2020.

\section{Generation of wapo-A1 wapo-B1 double mutants using CRISPR-Cas9}

To characterize better the function of WAPO1, we edited both homeologs of the tetraploid variety Kronos using CRISPR-Cas9 [37]. We designed one guide RNA between positions 494 and 512 from the starting ATG of the coding region (S1 Table) to induce double-strand breaks in the first exon of both WAPO-A1 and WAPO-B1. This guide RNA was then cloned into a vector which included the Cas9 gene and a GRF4-GIF1 chimera that increases wheat regeneration efficiency for Agrobacterium-mediated transformation [38]. Three independent $\mathrm{T}_{0}$ transgenic Kronos plants were obtained from the UCD Plant Transformation facility and were screened for mutations by next generation sequencing (NGS) and restriction enzyme digestion. For the NGS screen, we used primers g641-NGS-F and R that amplify both genomes (S1 Table) and analyzed the data using CRISgo (https://github.com/pinbo/CRISgo) as published before [39]. For the restriction enzyme screen, we used A-genome specific primer pair CAPSWAPO-A1-F and R1 and B-genome specific primer pair CAPS-WAPO-B1-F and R2 (S1 Table) followed by digestion with restriction enzyme $X \mathrm{cmI}$. The same primers and restriction enzyme digestion were used as Cleavage Amplified Polymorphism Sequence (CAPS) markers for subsequent generations.

\section{Generation of WAPO1 transgenic lines}

To validate the role of WAPO-A1 in the regulation of SNS, we generated transgenic plants expressing the WAPO-A1 gene driven by its native promoter. The genomic region of $W A P O-A 1$, which included $4.8 \mathrm{~kb}$ upstream of the start codon, the complete coding region with its intron, and $1.5 \mathrm{~kb}$ downstream of the stop codon, was cloned into the hygromycinresistance binary vector pLC41. Three different $W A P O-A 1$ alleles, each encoding a different WAPO-A1 protein (Table 1), were cloned and transformed into Kronos. The first genomic region of WAPO- $A^{m} 1$ was obtained from the BAC library of diploid T. monococcum ssp. monococcum accession DV92, henceforth TmDV92 (genome $\mathrm{A}^{\mathrm{m}}$ ) [18]. The WAPO- $\mathrm{A}^{\mathrm{m}} 1$ protein encoded by the TmDV92 allele differs from WAPO-A1 in polyploid wheat by three amino acids, but is otherwise similar to the $\mathrm{H} 3$ haplotype (Table 1). The second WAPO-A1 genomic

region was cloned from the BAC library of T. turgidum ssp. durum variety Langdon [19]. This construct, designated hereafter as LDN-C47, encodes the Wapo-A1d allele (H3 haplotype), which has no deletion in the promoter region and has the ancestral amino acids C47 and D384 (Table 1) 
The third construct, named LDN-F47, was created by site-directed mutagenesis of construct LDN-C47 using primers described in S1 Table. The change from the ancestral cysteine (C47) into a phenylalanine (F47), resulted in a protein identical to the one encoded by the Wapo-A $1 b$ allele in the $\mathrm{H} 2$ haplotype (Table 1 ). However, the LDN-F47 clone differs from the natural $\mathrm{H} 2$ genomic region by three SNPs in the promoter region and two in the first intron [10]. Table 1 presents a summary of the different WAPO-A1 constructs and their comparison with the endogenous Wapo-Ala allele (H1 haplotype) present in the transformed variety Kronos.

Constructs were transformed into Kronos using Agrobacterium-mediated transformation (EHA105) at the UC Davis Plant Transformation Facility as described before [38]. Primers listed in S1 Table were used to genotype the transgenic plants and confirm the presence of the transgene. Transgenic $T_{0}$ plants were advanced to $T_{1}$ and characterized in the greenhouse for heading date, SNS, and spike and floral morphology.

\section{Populations used to compare the effects of WAPO-A1 haplotypes in the field}

To compare the effect of the $\mathrm{H} 1$ and $\mathrm{H} 3$ haplotypes on SNS, we developed a population segregating for the $\mathrm{H} 1$ haplotype from Kronos and the $\mathrm{H} 3$ haplotype from the durum wheat variety Rusty, which carried the same Wapo-A1d allele as Langdon [10] (Table 1). From the Kronos $\mathrm{x}$ Rusty cross [40], $75 \mathrm{~F}_{2}$ plants were advanced to $\mathrm{F}_{4}$ by single seed descent (SDS) and genotyped for the WAPO-A1 haplotype using a molecular marker previously developed for the WAPO-A1 promoter deletion [10]. We selected eight heterozygous $\mathrm{F}_{4}$ plants (IDs $=12,19,51$, $55,69,113,120,128)$ to generate eight Heterozygous Inbred Families (HIFs). For each $F_{4: 5}$ HIF, we selected two homozygous sister lines $\left(\mathrm{F}_{4: 6}\right)$-one fixed for $\mathrm{H} 1$ and the other fixed for H3.

The $\mathrm{F}_{4: 6}$ grains were used for two field trials conducted at UC Davis experimental field station that were planted in October and harvested in June of the next year. The experiments are referred to using their harvest years (2020 and 2021). Both field experiments were organized in a split plot randomized complete block design (RCBD) with 10 replications, using the eight HIFs as main plots, and sister lines fixed for $\mathrm{H} 1$ and $\mathrm{H} 3$ haplotypes as subplots. For the 2020 experiment, we used single rows as experimental units and measured 15 spikes per row. For the 2021 experiment, we used small plots $\left(3\right.$ rows $\left.=1.86 \mathrm{~m}^{2}\right)$ as experimental units and measured 12 spikes per plot for SNS determinations. In 2021, we also measured heading date as the time from the first rain after sowing to the time when half of the plants in the plot have headed, grain number per spike (each replication was the average of 3 spikes), and total grain yield in $\mathrm{kg} / \mathrm{ha}$ (small plots were harvested with a Wintersteiger Combine).

To compare the effect of $\mathrm{H} 2$ relative to $\mathrm{H} 1$, we developed NILs segregating for these haplotypes in both tetraploid and hexaploid wheat. The tetraploid NILs were developed by introgressing the WAPO-A1 H2 haplotype from UC Davis common wheat breeding line UC1110 (also referred to as CAP1) into tetraploid wheat variety Kronos using marker assisted backcrossing. After three backcrosses with Kronos, we self-pollinated the $\mathrm{BC}_{3}$ plants, and selected $\mathrm{BC}_{3} \mathrm{~F}_{2}$ plants homozygous for the $\mathrm{H} 1$ and $\mathrm{H} 2$ haplotypes. The derived $\mathrm{BC}_{3} \mathrm{~F}_{3}$ grains were sown at UC Davis in November 2020 in a completely randomized design (CRD) with nine replications, using 1-m rows as experimental units (10 spikes were measured per row and averaged). The hexaploid NILs were developed by backcrossing the $\mathrm{H} 2$ haplotype five times into the hexaploid line GID4314513 (H1) from the CIMMYT high-biomass program. We selected a highbiomass line as recurrent parent to increase the probability that increases in SNS and GNS would be translated into increases in total grain yield. We developed $\mathrm{BC}_{5} \mathrm{~F}_{3}$ homozygous NILs 
using GID4314513 as recurrent parent, and compared them in the field at UC Davis in 2021 using an RCBD with 10 replications and 3-row small plots $\left(1.86 \mathrm{~m}^{2}\right)$ as experimental units. We measured 4 spikes per plot (subsamples) and harvested the small plots with a combine.

\section{Gene expression studies}

To test if the differences in SNS between $\mathrm{H} 3$ and $\mathrm{H} 1$ were associated with different levels of $W A P O-A 1$ expression, we compared the transcript levels of this gene in developing spikes of homozygous $\mathrm{H} 1$ and $\mathrm{H} 3$ sister lines derived from HIF \#120. WAPO-A1 transcript levels were determined by qRT-PCR using A-genome-specific primers WAPO-A1-RT-F2 and WAPO1-A1-RT-R2 and PCR conditions described in our previous study [10]. Reactions were performed on an ABI 7500 Fast Real-Time PCR System (Applied Biosystems) using Fast SYBR GREEN Master Mix. Transcript levels were expressed as fold-ACTIN levels using the $2^{\Delta C T}$ method.

Plants were grown in $1.4 \mathrm{~L}$ cones placed in CONVIRON growth chambers under $16 \mathrm{~h}$ light at $22^{\circ} \mathrm{C}$ (330 mol intensity) and $8 \mathrm{~h}$ darkness at $17^{\circ} \mathrm{C}$ for $25-30$ days. For each replicate, we pooled 3-8 developing spikes from the main tiller when the lemma primordia were visible (W3.25) in the first and second experiments or when the floret primordia were present (W3.5) in the third experiment. Spike developmental stages are based on the Waddington scale [41]. We analyzed the first two experiments at W3.25 together using experiment as block, and the third experiment at W3.5 separately.

To compare the transcript levels of class-B, -C and -E MADS-box genes in Kronos and the wapo1 mutant, we extracted RNA from developing spikes at the stamen primordia stage $(\sim \mathrm{W} 4.0)$. A total of 12 developing spikes were pooled per replicate. Plants were grown in onegallon pots in a CONVIRON growth chamber under similar conditions as described above. RNA extraction and expression analyses were done as described previously [42]. Transcript levels of WAPO-A1 in the transgenic plants were conducted in a similar way, but developing spikes were harvested at stage W3.5 and 5-6 developing spikes were pooled per replicate.

For each transgenic construct, we selected one sister line without the transgene (WT), one with weak spike phenotype (e.g. increased SNS) and one with strong spike phenotypes (naked pistils and small terminal spikelet) and analyzed WAPO-A1 transcript levels using primers WAPO-A1-RT-F2 and WAPO-A1-RT-R2 (S1 Table). We grew 24-36 $\mathrm{T}_{2}$ progeny from each of the selected $\mathrm{T}_{1}$ plants in a growth chamber and extracted RNA from pools of 6-9 developing spikes at the floret primordia stage (W3.5).

\section{In situ hybridization}

We performed in situ RNA hybridization following the protocol described previously [21]. Tissues were obtained from developing spikes of diploid T. monococcum (accession PI 167615) and tetraploid wheat cultivar Kronos. cDNAs obtained from T. monococcum and Kronos were used to amplify WAPO1 genes for the in vitro transcription reaction. We designed wheat Agenome specific primers appended with promoter sequences of T3 and T7 (S1 Table). Probes were synthesized using T3 (sense probe) or T7 (antisense probe) RNA Polymerase (Roche) and labelled with Digoxigenin-11-UTP (Roche). The forward primer P3-WM-APO1-T3- $\mathrm{F}_{1400}$ starts 57 bp upstream of the stop codon and two alternative reverse primers P4-WM-APO1-T7- $\mathrm{R}_{1649}$ and P5-WM-APO1-T7- $\mathrm{R}_{1843}$ end in the 3' UTR and include a total of 266 and 458 bp respectively ( $\mathrm{S} 1 \mathrm{Table}$ ). The $\mathrm{P} 3-\mathrm{P} 4$ and $\mathrm{P} 3-\mathrm{P} 5$ probes showed the same specificity. The color reaction was stopped at 48 or 72 hours, and images were taken by Nikon Ti Microscope equipped with a DS-Fi2-U3 camera. 


\section{Statistical analyses}

The interaction between $W A P O-A 1$ and $W A P O-B 1$ was tested using a 2 x 2 factorial ANOVA using homeologs as factors and alleles (WT and CRISPR mutants) as levels. The four possible homozygous classes-WT, wapo-A1, wapo-B1, and wapo1 double mutant-were selected from the $\mathrm{F}_{2}$ progeny of an $\mathrm{F}_{1}$ plant from the cross wapo1 x Kronos WT without the CRISPR-Cas 9 transgene.

For the transgenic lines TmDV92, LDN-C47, and LDN-F47, we generated three to five different transgenic events. Since the number of $\mathrm{T}_{1}$ non-transgenic sister lines was small for each event, we pooled the non-transgenic sister lines from the different events generated with the same construct. We then compared the different transgenic events with the pooled non-transgenic lines with the same construct using Dunnett tests. All statistical analyses were performed with SAS version 9.4. Homogeneity of variance was tested using the Levene's test and normality of residual using the Shapiro-Wilks test as implemented in SAS v9.4. If necessary, data was transformed to restore the assumptions of the ANOVA. Data and descriptive statistics are provided in S1 Data file in Excel format, with data for the same figures and/or supplemental tables organized within a spreadsheet named with letters A to $\mathrm{H}$.

\section{Supporting information}

S1 Table. Primers used in this study. (DOCX)

S2 Table. $\mathrm{F}_{2}$ CRISPR loss-of-function mutants WAPO-A1 1 WAPO-B1 factorial ANOVA for spikelet number per spike (SNS), heading date and leaf number of the main tiller at heading. (DOCX)

S3 Table. ANOVAs for 2020 and 2021 experiments testing the effect of $\mathrm{H} 3$ and $\mathrm{H} 1$ haplotypes on SNS, grain number per spike (GNS), grain yield, thousand kernel weight, and heading date.

(DOCX)

S4 Table. ANOVAs for 2021 experiment to evaluate the effect of WAPO-A1 haplotype $\mathrm{H} 2$ introgressed into tetraploid Kronos and hexaploid high-biomass line GID4314513. (DOCX)

S1 Fig. Expression analysis of WAPO-A1 and WAPO-B1 homeologs in developing spikes. (DOCX)

S2 Fig. Strongest floral abnormalities in Kronos plants transformed with WAPO-A1 genomic regions of $\mathrm{LDN}-\mathrm{F} 47-2$.

(DOCX)

S3 Fig. In situ hybridization of WAPO1 probe amplified from T. monococcum in developing spikes of diploid wheat $T$. monococcum.

(DOCX)

S1 Data. Data A. Supporting data for Fig 1A-1C. Spikelet number per spike (SNS) in lines derived from Kronos EMS loss-of-function mutant K4222 for wapo-A1. Data B. Supporting data for Fig 2. Spikelet number per spike (SNS) and days to heading (DTH) in transgenic $\mathrm{T}_{1}$ plants (different events) relative to pooled non-transgenic plants. Data C. Supporting data for Fig 3A-3D. Frequency of floral abnormalities in 14 Kronos plants with loss-of-function CRISPR mutations in both wapo-A1 and wapo-B1 (32 spikelets, 91 florets). Data D. 
Supporting data for Fig 3E-3G. Expression of MADS-box genes involved in floral development in WT Kronos and wapo1-null (wapo-A1 wapo-B1) CRISPR truncation mutants. Data E. Supporting data for Fig 5. WAPO-A1 transcript levels and fertility in Kronos transgenic plants transformed with WAPO-A1 driven by its natural promoter. Data F. Supporting data for Fig 7A-7D and S3 Table. Split plot randomized complete block design field experiment testing the effect of WAPO-A1 H1 and H3 haplotypes. Data G. Supporting Data for Fig 7E and 7F. Expression of WAPO-A1 in homozygous $\mathrm{H} 1$ and $\mathrm{H} 3$ sister lines derived from HIF \#120. Data H. Supporting data for Fig 8 and S4 Table. Effect of WAPO-A1 haplotype $\mathrm{H} 2$ introgressed into tetraploid Kronos (CRD, 9 reps.) and high-biomass hexaploid line GID4314513 (RCBD, 10 blocks) in field experiments performed in 2021.

(XLSX)

\section{Acknowledgments}

We thank Mariana Padilla and Oswaldo Chicaiza for excellent technical assistance in designing, managing, and collecting all field experiment data and to Juan Debernardi for reviewing the manuscript and valuable suggestions. We also thank André Schönhofen, Xiaoqin Zhang, and Priscilla Glenn for their help with the introgression of the $\mathrm{H} 2$ haplotype from common wheat into Kronos.

\section{Author Contributions}

Conceptualization: Jorge Dubcovsky.

Data curation: Jorge Dubcovsky.

Formal analysis: Saarah Kuzay, Huiqiong Lin, Daniel P. Woods, Jorge Dubcovsky.

Funding acquisition: Jorge Dubcovsky.

Investigation: Saarah Kuzay, Huiqiong Lin, Chengxia Li, Daniel P. Woods, Tianyu Lan, Maria von Korff.

Methodology: Saarah Kuzay, Huiqiong Lin, Chengxia Li, Daniel P. Woods, Jorge Dubcovsky.

Project administration: Jorge Dubcovsky.

Resources: Saarah Kuzay, Huiqiong Lin, Chengxia Li, Shisheng Chen, Junli Zhang.

Supervision: Chengxia Li, Daniel P. Woods, Maria von Korff, Jorge Dubcovsky.

Visualization: Saarah Kuzay, Huiqiong Lin, Chengxia Li, Daniel P. Woods, Jorge Dubcovsky.

Writing - original draft: Saarah Kuzay.

Writing - review \& editing: Saarah Kuzay, Huiqiong Lin, Chengxia Li, Shisheng Chen, Daniel P. Woods, Junli Zhang, Tianyu Lan, Maria von Korff, Jorge Dubcovsky.

\section{References}

1. FAOSTAT. http://www.fao.org/faostat/en/\#data: Food and Agriculture Organization (FAO) of the United Nations; 2017.

2. Grassini $P$, Eskridge KM, Cassman KG. Distinguishing between yield advances and yield plateaus in historical crop production trends. Nat Commun. 2013; 4:2918. https://doi.org/10.1038/ncomms3918 WOS:000329396500004. PMID: 24346131

3. Slafer GA. Genetic basis of yield as viewed from a crop physiologist's perspective. Ann Appl Biol. 2003; 142(2):117-28. https://doi.org/10.1111/j.1744-7348.2003.tb00237.x WOS:000182916100002. 
4. Zhang JL, Gizaw SA, Bossolini E, Hegarty J, Howell T, Carter AH, et al. Identification and validation of QTL for grain yield and plant water status under contrasting water treatments in fall-sown spring wheats. Theor Appl Genet. 2018; 131(8):1741-59. https://doi.org/10.1007/s00122-018-3111-9 WOS:000439137500011. PMID: 29767279

5. Ward BP, Brown-Guedira G, Kolb FL, Van Sanford DA, Tyagi P, Sneller CH, et al. Genome-wide association studies for yield-related traits in soft red winter wheat grown in Virginia. PLoS One. 2019; 14(2): e0208217. https://doi.org/10.1371/journal.pone.0208217 WOS:000459709100008. PMID: 30794545

6. Boeven PHG, Longin CFH, Leiser WL, Kollers S, Ebmeyer E, Wurschum T. Genetic architecture of male floral traits required for hybrid wheat breeding. Theor Appl Genet. 2016; 129(12):2343-57. https:// doi.org/10.1007/s00122-016-2771-6 WOS:000389254700010. PMID: 27553082

7. Muqaddasi QH, Brassac J, Koppolu R, Plieske J, Ganal MW, Roder MS. TaAPO-A1, an ortholog of rice ABERRANT PANICLE ORGANIZATION 1, is associated with total spikelet number per spike in elite European hexaploid winter wheat (Triticum aestivum L.) varieties. Sci Rep-Uk. 2019; 9:ARTN 13853. https://doi.org/10.1038/s41598-019-50331-9 WOS:000487586600044. PMID: 31554871

8. Voss-Fels KP, Keeble-Gagnere G, Hickey LT, Tibbits J, Nagornyy S, Hayden MJ, et al. High-resolution mapping of rachis nodes per rachis, a critical determinant of grain yield components in wheat. Theor Appl Genet. 2019; 132(9):2707-19. https://doi.org/10.1007/s00122-019-03383-4 WOS:000484527400018. PMID: 31254025

9. Wurschum T, Leiser WL, Langer SM, Tucker MR, Longin CFH. Phenotypic and genetic analysis of spike and kernel characteristics in wheat reveals long-term genetic trends of grain yield components. Theor Appl Genet. 2018; 131(10):2071-84. https://doi.org/10.1007/s00122-018-3133-3 WOS:000445158000004. PMID: 29959471

10. Kuzay S, Xu Y, Zhang J, Katz A, Pearce S, Su Z, et al. Identification of a candidate gene for a QTL for spikelet number per spike on wheat chromosome arm 7AL by high-resolution genetic mapping. Theor Appl Genet. 2019; 132:2689-705. https://doi.org/10.1007/s00122-019-03382-5 PMID: 31254024.

11. Ikeda K, Ito M, NagasawaO N, Kyozuka J, Nagato Y. Rice ABERRANT PANICLE ORGANIZATION 1 encoding an F-box protein, regulates meristem fate. Plant J. 2007; 51(6):1030-40. https://doi.org/10. 1111/j.1365-313X.2007.03200.x WOS:000249424200008. PMID: 17666027

12. Ikeda $\mathrm{K}$, Nagasawa $\mathrm{N}$, Nagato $\mathrm{Y}$. ABERRANT PANICLE ORGANIZATION 1 temporally regulates meristem identity in rice. Dev Biol. 2005; 282(2):349-60. https://doi.org/10.1016/j.ydbio.2005.03.016 WOS:000230005900006. PMID: 15950602

13. Levin JZ, Meyerowitz EM. Ufo-an Arabidopsis gene involved in both floral meristem and floral organ development. Plant Cell. 1995; 7(5):529-48. https://doi.org/10.1105/tpc.7.5.529 WOS: A1995RA41700005. PMID: 7780306

14. Chae E, Tan QKG, Hill TA, Irish VF. An Arabidopsis F-box protein acts as a transcriptional co-factor to regulate floral development. Development. 2008; 135(7):1235-45. https://doi.org/10.1242/dev.015842 WOS:000254917500004. PMID: 18287201

15. Ikeda-Kawakatsu K, Maekawa M, Izawa T, Itoh JI, Nagato Y. ABERRANT PANICLE ORGANIZATION 2/RFL, the rice ortholog of Arabidopsis $L E A F Y$, suppresses the transition from inflorescence meristem to floral meristem through interaction with APO1. Plant J. 2012; 69(1):168-80. https://doi.org/10.1111/j. 1365-313X.2011.04781.x WOS:000298357900015. PMID: 21910771

16. Krasileva KV, Vasquez-Gross HA, Howell T, Bailey P, Paraiso F, Clissold L, et al. Uncovering hidden variation in polyploid wheat. Proc Natl Acad Sci U S A. 2017; 114(6):E913-E21. https://doi.org/10.1073/ pnas.1619268114 PMID: 28096351.

17. Falconer DS. Introduction to quantitative genetics. Edinburgh: Oliver \& Boyd 1964.

18. Lijavetzky D, Muzzi G, Wicker T, Keller B, Wing R, Dubcovsky J. Construction and characterization of a bacterial artificial chromosome (BAC) library for the A genome of wheat. Genome. 1999; 42(6):117682. PMID: 10659785.

19. Cenci A, Chantret N, Kong X, Gu Y, Anderson OD, Fahima T, et al. Construction and characterization of a half million clone BAC library of durum wheat (Triticum turgidum ssp. durum). Theor Appl Genet. 2003; 107(5):931-9. ISI:000185117600020. https://doi.org/10.1007/s00122-003-1331-z PMID: 12830387

20. Schilling S, Kennedy A, Pan S, Jermiin LS, Melzer R. Genome-wide analysis of MIKC-type MADSbox genes in wheat: pervasive duplications, functional conservation and putative neofunctionalization New Phytol. 2020; 225(1):511-29. https://doi.org/10.1111/nph.16122 PMID: 31418861.

21. Zhong J, van Esse GW, Bi X, Lan T, Walla A, Sang Q, et al. INTERMEDIUM-M encodes an HvAP2L$\mathrm{H} 5$ ortholog and is required for inflorescence indeterminacy and spikelet determinacy in barley. Proc Natl Acad Sci U S A. 2021; 118(8):e2011779118. https://doi.org/10.1073/pnas.2011779118 WOS:000621797000020. PMID: 33593903 
22. Lee I, Wolfe DS, Nilsson O, Weigel D. A LEAFY co-regulator encoded by UNUSUAL FLORAL ORGANS. Curr Biol. 1997; 7(2):95-104. https://doi.org/10.1016/s0960-9822(06)00053-4 WOS: A1997WH22600022. PMID: 9016705

23. Hepworth SR, Klenz JE, Haughn GW. UFO in the Arabidopsis inflorescence apex is required for floralmeristem identity and bract suppression. Planta. 2006; 223(4):769-78. https://doi.org/10.1007/s00425005-0138-3 WOS:000236965600014. PMID: 16244866

24. Wagner D, Sablowski RWM, Meyerowitz EM. Transcriptional activation of APETALA1 by LEAFY. Science. 1999; 285(5427):582-4. https://doi.org/10.1126/science.285.5427.582 WOS:000081609500037. PMID: 10417387

25. Grandi V, Gregis V, Kater MM. Uncovering genetic and molecular interactions among floral meristem identity genes in Arabidopsis thaliana. Plant J. 2012; 69(5):881-93. https://doi.org/10.1111/j.1365313X.2011.04840.x WOS:000300696800012. PMID: 22040363

26. Li C, Lin H, Chen A, Lau M, Jernstedt J, Dubcovsky J. Wheat VRN1, FUL2 and FUL3 play critical and redundant roles in spikelet development and spike determinacy. Development. 2019; 146(14): dev175398. https://doi.org/10.1242/dev.175398 WOS:000478027300017. PMID: 31337701

27. Li K, Debernardi JM, Li C, Lin H, Zhang C, Dubcovsky J. Interactions between SQUAMOSA and SVP MADS-box proteins regulate meristem transitions during wheat spike development. The Plant Cell. 2021; 33(12):3621-44. https://doi.org/10.1093/plcell/koab243 PMID: 34726755

28. Lamb RS, Hill TA, Tan QKG, Irish VF. Regulation of APETALA3 floral homeotic gene expression by meristem identity genes. Development. 2002; 129(9):2079-86. WOS:000175694400003. PMID: 11959818

29. Busch MA, Bomblies K, Weigel D. Activation of a floral homeotic gene in Arabidopsis. Science. 1999; 285(5427):585-7. https://doi.org/10.1126/science.285.5427.585 PMID: 10417388.

30. Lohmann JU, Hong RL, Hobe M, Busch MA, Parcy F, Simon R, et al. A molecular link between stem cell regulation and floral patterning in Arabidopsis. Cell. 2001; 105(6):793-803. https://doi.org/10.1016/ s0092-8674(01)00384-1 WOS:000169375100012. PMID: 11440721

31. Krizek BA, Meyerowitz EM. The Arabidopsis homeotic genes APETALA3 and PISTILLATA are sufficient to provide the B class organ identity function. Development. 1996; 122(1):11-22. WOS: A1996TY51600002. PMID: 8565821

32. Wilkinson MD, Haughn GW. UNUSUAL FLORAL ORGANS controls meristem identity and organ primordia fate in Arabidopsis. Plant Cell. 1995; 7(9):1485-99. https://doi.org/10.1105/tpc.7.9.1485 PMID: 12242408.

33. Boden SA, Cavanagh C, Cullis BR, Ramm K, Greenwood J, Jean Finnegan E, et al. Ppd-1 is a key regulator of inflorescence architecture and paired spikelet development in wheat. Nat Plants. 2015;1. https://doi.org/10.1038/nplants.2014.16 PMID: 27246757

34. Poursarebani N, Seidensticker T, Koppolu R, Trautewig C, Gawronski P, Bini F, et al. The genetic basis of composite spike form in barley and 'Miracle-Wheat'. Genetics. 2015; 201(1):155-65. https://doi.org/ 10.1534/genetics.115.176628 WOS:000361206400013. PMID: 26156223

35. Wolde GM, Mascher M, Schnurbusch T. Genetic modification of spikelet arrangement in wheat increases grain number without significantly affecting grain weight. Mol Genet Genomics. 2019; 294:457-68. https://doi.org/10.1007/s00438-018-1523-5 PMID: 30591960.

36. Glenn P, Zhang J, Brown-Guedira G, DeWitt N, Cook JP, Li K, et al. Identification and characterization of a natural polymorphism in $F T$-A2 associated with increased number of grains per spike in wheat. Theor Appl Genet. 2021; https://doi.org/10.1007/s00122-021-03992-y PMID: 34825926

37. Jinek M, Chylinski K, Fonfara I, Hauer M, Doudna JA, Charpentier E. A programmable dual-RNAguided DNA endonuclease in adaptive bacterial immunity. Science. 2012; 337(6096):816-21. https:// doi.org/10.1126/science.1225829 WOS:000307535600036. PMID: 22745249

38. Debernardi JM, Tricoli DM, Ercoli MF, Hayta S, Ronald P, Palatnik JF, et al. A GRF-GIF chimeric protein improves the regeneration efficiency of transgenic plants. Nat Biotechnol. 2020; 38(11):1274-9. https:// doi.org/10.1038/s41587-020-0703-0 PMID: 33046875.

39. Connelly JP, Pruett-Miller SM. CRIS. py: A versatile and high-throughput analysis program for CRISPRbased genome editing. Sci Rep-Uk. 2019; 9:4194. https://doi.org/10.1038/s41598-019-40896-w WOS:000460922200030. PMID: 30862905

40. Simons K, Abate Z, Chao SM, Zhang WJ, Rouse M, Jin Y, et al. Genetic mapping of stem rust resistance gene Sr13 in tetraploid wheat (Triticum turgidum ssp. durum L.). Theor Appl Genet. 2011; 122 (3):649-58. https://doi.org/10.1007/s00122-010-1444-0 ISI:000286598900018. PMID: 20857083

41. Waddington SR, Cartwright PM, Wall PC. A quantitative scale of spike initial and pistil development in barley and wheat. Ann Bot-London. 1983; 51(1):119-30. WOS:A1983QC01200013. 
42. Shaw LM, Li CX, Woods DP, Alvarez MA, Lin HQ, Lau MY, et al. Epistatic interactions between PHOTOPERIOD1, CONSTANS1 and CONSTANS2 modulate the photoperiodic response in wheat. PLoS Genet. 2020; 16(7):e1008812. https://doi.org/10.1371/journal.pgen.1008812 WOS:000552626900001. PMID: 32658893 